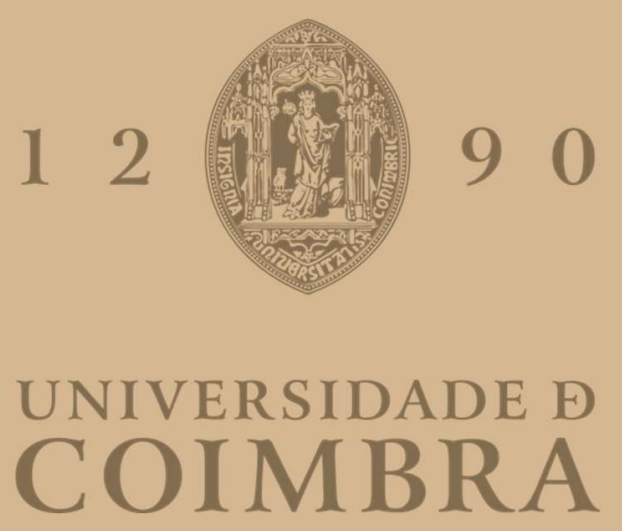

Mónica Alexandra Correia Sobral

\title{
Association BetWeEn CoRTiCAL ThickNess AND ANXIETY MEASURES \\ A SCOPING REVIEW
}

Dissertação no âmbito do Mestrado Integrado em Psicologia, área de especialização em Intervenções Cognitivo-Comportamentais nas Perturbações Psicológicas e Saúde, orientada pela Professora Doutora Helena Teresa da Cruz Moreira e Doutora Ana Ganho Ávila Costa e apresentada à Faculdade de Psicologia e de Ciências da Educação da Universidade de Coimbra.

Julho de 2020 


\title{
ASSOCIATION BETWEEN CORTICAL \\ THICKNESS AND ANXIETY MEASURES: \\ A Scoping Review
}

\author{
Mónica Alexandra Correia Sobral
}

\begin{abstract}
Dissertação no âmbito do Mestrado Integrado em Psicologia, área de especialização em Intervenções Cognitivo-Comportamentais nas Perturbações Psicológicas e Saúde, orientada pela Professora Doutora Helena Teresa da Cruz Moreira e Doutora Ana Ganho Ávila Costa e apresentada à Faculdade de Psicologia e de Ciências da Educação da Universidade de Coimbra.
\end{abstract}

Julho de 2020

12

90

UNIVERSIDADE E

COIMBRA 



\section{Acknowledgments}

I would like to thank the following people for the assistance and companion provided with this endeavor, without whom I would have not been able to complete it.

To Professor and Supervisor Ana Ganho, for all the insights, knowledge, encouragement, patience and for always going the extra mile to make sure I could achieve what I had in mind. You have been a true mentor and guided me so positively. To Professor and Supervisor Helena Moreira, for your support, dedication, patience and guidance during this year as well.

To Sara Morgado, for her vital part in this process and for taking the time to help me complete the research. To Raquel Guiomar, whose insight, knowledge and stimulating discussions guided me multiple times. Thank you for all the enthusiasm and confidence placed in me in the past two years.

To Catarina and Inês Caçador, for being my companions over this year and for all the dedication and friendship you have shown me.

To Carla, Inês and Marta for their unconditional support these past five years and for the many memories we built together. To Marta in specifically for being the strength I needed when everything was new. To Adriana, who made sure from the first moment I felt like I belonged and has been by my side ever since, cheering me on.

To Ricardo, thank you for putting up with me for hours on end and for being my safe place. To Eugénio and Ana Guedes, for welcoming me with arms open into their family and all the opportunities you have given me.

To my parents, for educating and preparing me for this moment and for all the sacrifices and support you have given me throughout my life.

This was a year of strong emotions. Thank you all for being a part of it. 


\section{Resumo}

Revisão da Literatura: As perturbações de ansiedade são uma das perturbações mais prevalentes e interferentes. A investigação acerca dos correlatos neurais da ansiedade, como a espessura cortical, pode proporcionar uma melhor compreensão dos mecanismos neurais subjacentes a diferenças individuais em perfis distintos de sintomas de ansiedade e vulnerabilidade a perturbações de ansiedade. Objetivo: Esta Scoping Review pretende sumariar a literatura publicada desde 2004 no contexto de estudos pré-clínicos, clínicos, e de ciência básica, no que diz respeito à associação entre espessura cortical e medidas de ansiedade em uso, de forma a determinar o papel da espessura cortical como um fator de vulnerabilidade ao desenvolvimento de perturbações de ansiedade. Métodos: A revisão seguiu as orientações da metodologia de Arksey e O’Malley. As pesquisas foram realizadas em bases de dados eletrónicas (PubMed, PsycINFO e PsycARTICLES, e Web of Science) e lista de referências de estudos-chave. Dois investigadores avaliaram independentemente os resumos e texto completo dos artigos de acordo com os critérios de inclusão, extraíram e organizaram os dados. Sínteses quantitativas (i.e., numéricas) e qualitativas (i.e., abordagem narrativa) foram realizadas de modo a caracterizar os estudos incluídos e a encontrar lacunas no conhecimento. Resultados: Um total de 17 artigos foi incluído na revisão final. Todas as publicações reportaram estudos transversais, com a maioria $(n=16)$ a adotar abordagens surfacebased para a estimativa de espessura cortical. De um modo geral, associações estatisticamente significativas entre espessura cortical e medidas/processos de ansiedade foram encontradas, para instrumentos de autorrelato (e.g., Inventário de Estado-Traço de Ansiedade), medidas psicofisiológicas (e.g., respostas galvânicas da pele), e neuroimagem funcional. Conclusões: Diferenças na espessura cortical em diversas regiões cerebrais encontraram-se associadas a várias medidas e processos subjacentes a ansiedade. Regiões de potencial interesse incluem o córtex orbitofrontal medial, o córtex pré-frontal ventromedial, a insula, as áreas temporoparietais, e o córtex cingulado anterior. Alterações na espessura cortical regional podem constituir um fator de vulnerabilidade ao desenvolvimento de perturbações de ansiedade, embora investigação adicional acerca desta associação seja necessária, nomeadamente com populações saudáveis ou através de desenhos longitudinais. Não obstante, a possibilidade de uma relação bidirecional não pode ser excluída.

Palavras-chave: espessura cortical, ansiedade, perturbações de ansiedade, medo, condicionamento, avaliação, medidas de ansiedade, vulnerabilidade 


\begin{abstract}
Background: Anxiety disorders are amongst the most common and interfering disorders. Research on the neural correlates of anxiety, such as cortical thickness, may provide a better understanding of the neural mechanisms underlying individual differences in what concerns distinctive profiles of anxiety symptoms and vulnerability to anxiety disorders. Objective: This scoping review aims to summarize the literature published since 2004 in the context of pre-clinical, clinical, and basic science studies, concerning the association between cortical thickness and anxiety measures in use, to determine the role of cortical thickness as a vulnerability factor for the development of anxiety disorders. Design: Our review followed the guidelines of the Arksey and O'Malley methodology. Searches were conducted in electronic databases (PubMed, PsycINFO and PsycARTICLES, and Web of Science) and reference lists of key studies. Two researchers independently screened the abstracts and full-text articles according to the eligibility criteria, as well as extracted and charted the data. Quantitative (i.e., numerical) and qualitative (i.e., narrative approach) syntheses were conducted to characterize the included studies and find gaps in knowledge. Results: A total of 17 articles were included in the final review. All publications reported cross-sectional studies, with the majority ( $n=16)$ employing surface-based approaches to the measurement of cortical thickness. Overall, statistically significant associations between cortical thickness and measures/processes of anxiety were found, for self-report instruments (e.g., State-Trait Anxiety Inventory), psychophysiological measures (e.g., skin conductance reponses), and functional neuroimaging. Conclusions: Differences in cortical thickness across several brain regions were found to be associated with different measures and processes underlying anxiety. Regions of potential interest include the medial orbitofrontal cortex, the ventromedial prefrontal cortex, the insula, the temporoparietal areas, and the anterior cingulate cortex. Alterations in regional cortical thickness may be a vulnerability factor for the development of anxiety disorders, although more research into this association is needed, namely with healthy population or within longitudinal designs. Nonetheless, the possibility of a bidirectional relationship cannot be excluded.
\end{abstract}

Keywords: cortical thickness, anxiety, anxiety disorders, fear, fear conditioning, assessment, anxiety measures, vulnerability 


\section{Table of Contents}

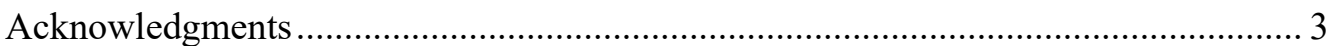

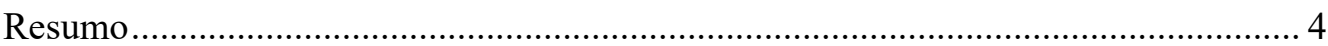

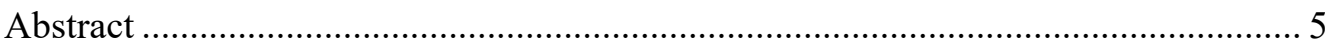

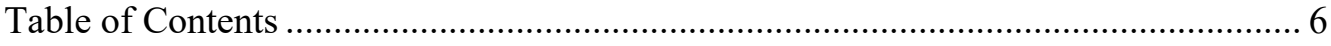

Association Between Cortical Thickness and Anxiety Measures: A Scoping Review...7

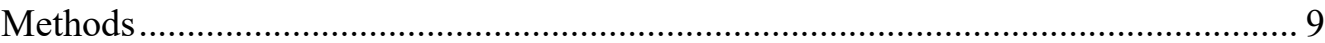

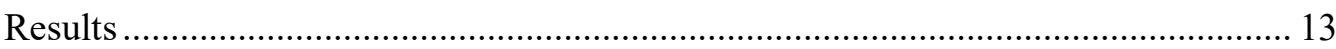

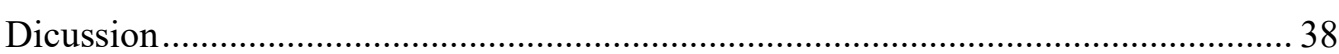

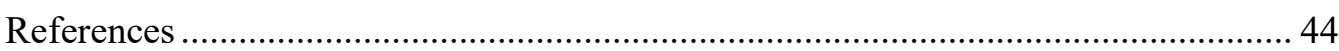

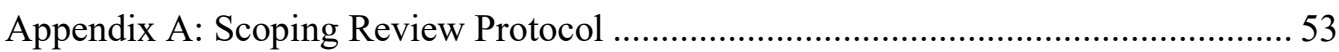

Appendix B: Identified Key Terms Across the Databases ............................................. 62

Appendix C: Final Search Strategy for the PsycINFO Database ...................................63

Appendix D: Joanna Briggs Institute Critical Appraisal Checklist for Analytical Cross-

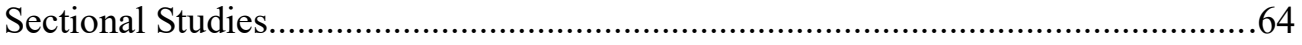

Appendix E: Critical Appraisal of Individual Sources of Evidence ................................ 65 


\section{Association Between Cortical Thickness and Anxiety Measures: A Scoping Review}

Anxiety disorders are amongst the most prevalent psychiatric disorders worldwide, with one out of fourteen people fulfilling the diagnostic criteria across their lifespan (Baxter et al., 2013). According to the Diagnostic and Statistical Manual of Mental Disorders (5th ed.; DSM-5; American Psychiatric Association [APA], 2013), anxiety disorders include generalized anxiety disorder (GAD), panic disorder (PD), specific phobias (SP), agoraphobia, social anxiety disorder (social phobia; SAD), separation anxiety disorder, selective mutism, substance/medication-induced anxiety disorder, and anxiety disorder due to another medical condition. It is important to note that obsessive-compulsive disorder (OCD), acute stress disorder, and posttraumatic stress disorder (PTSD) are no longer considered anxiety disorders by DSM-5 (APA, 2013).

Anxiety disorders are an excessive reaction of fear or anxiety, which are common human reactions with an adaptive function, signaling danger and helping to become more alert and prepared. Although there is a significant overlap between fear and anxiety, the two can be distinguished. For instance, fear entails a response to an immediate and identifiable threat. Alternatively, anxiety comprehends a more prolonged state of apprehension and tension to uncertain future events (Duval et al., 2015). Nevertheless, fear conditioning is able to produce behavioral symptoms underlying anxiety disorders (e.g., avoidance; Graham \& Milad, 2011).

Anxiety disorders are characterized by cognitive, behavioral, somatic, and emotional components (APA, 2013). There are several measures that attempt to assess the distinctive fear and anxiety components, namely: clinical self-report measures (e.g., Beck Anxiety Inventory [BAI; Beck et al., 1988], The State-Trait Anxiety Inventory [STAI; Spielberger et al., 1983]), psychophysiological measures (e.g., galvanic skin response, heart rate variability), functional neuroimaging (e.g., functional magnetic resonance imaging [fMRI], electroencephalography), neuroendocrine measures (e.g., cortisol levels), behavioral measures (e.g., reaction time), genetic (e.g., 5-HTTLPR), and epigenetic measures (e.g., serotonin pattern of methylation).

Fear conditioning processes are one of the mechanisms by which the development and maintenance of pathological anxiety is thought to occur (Lonsdorf et al., 2017). Abnormal fear conditioning processes, namely fear acquisition and conditioned fear-generalization, have been found to be associated with the pathogenesis of anxiety disorders (Tinoco-González et al., 2015). The fear response acquisition and extinction can be studied using experimental classical conditioning procedures, including fear acquisition, fear extinction, extinction recall and return of fear manipulations (e.g., renewal). However, the risk factors for the development of anxiety disorders and their interaction is not yet clear due to the complex combination of biopsychosocial factors. 
In an attempt to unveil the complexity of the fear response and anxiety disorders, translational neuroscience research (e.g., neuroimaging) has focused on the identification of perturbations in the brain structure, as well as on the determination of altered brain circuits that lay behind anxiety disorders, to inform and predict treatment response and guide the development of new treatments (Gold et al., 2017; Shin \& Liberzon, 2010). In this context, research has been exploring the neural biomarkers of anxiety and its related processes. A neural biomarker is "any measurable indicator of functional brain activity or morphological change of a disease or behavior that could be correlated with a single aspect of the disease process" (Donzuso et al., 2014, p. 505).

Several indices of structural changes in the brain are currently under study as potential neural biomarkers of anxiety disorders. In the last years, cortical grey matter has drawn researchers' particular interest. Cortical grey matter is defined as the grey matter contained between the greywhite interface and the pia mater (Hanford et al., 2016; Winkler et al., 2010). Its integrity can be evaluated through measures of volume, surface area, and thickness (Hanford et al., 2016). Grey matter volume is a composite of cortical surface area (SA) and cortical thickness (CT), with these two latter indices measuring the columnar organization of the cortex (Hanford et al., 2016; Panizzon et al., 2009). However, cortical SA and CT are distinct, both globally and regionally (Hanford et al., 2016; Panizzon et al., 2009; Winkler et al., 2010). Cortical SA is associated with the number of cortical columns ${ }^{1}$, while CT represents the number of cells within a column (Hanford et al., 2016; Panizzon et al., 2009; Rakic, 1988).

$\mathrm{CT}$ is commonly defined as the distance between two corresponding points on the pial and the white matter boundaries of the neocortex (Das et al., 2009). The thickness of the human cerebral cortex has an overall average of approximately $2.5-2.8 \mathrm{~mm}$, usually varying between 1 and $5 \mathrm{~mm}$ (Makris et al., 2006). CT can be a useful biomarker, as it may offer an adequate understanding of disease progression, the identification of related brain regions, and possibly support clinical diagnosis and clinical decision regarding treatment options (Hutton et al., 2008). Methods using CT seem to be sensitive in detecting structural abnormalities (Hutton et al., 2009), allowing to explore the structural correlates of anxiety as vulnerability factors (see, for example, the automated method developed by Fischl and Dale [2000], to accurately measure CT). However, because measurements of CT are based on different definitions and methods across studies, the field is lacking between studies comparisons (Das et al., 2009).

Changes in CT have been found in normal, neurodegenerative development (e.g., Singh et al., 2006), and psychiatric disorders, such as major depressive disorder (MDD; for a review, see Suh et al., 2019) and anxiety disorders (e.g., Kang et al., 2017; Syal et al., 2012). In what concerns

\footnotetext{
${ }^{1}$ The cerebral cortex comprehends a sheet of neurons in organized layers intersected by columns (Rakic, 1988). The functional unit of the cortex is the cortical column, which consists of an array of iterative neuronal groups (Rakic, 2007).
} 
anxiety disorders, differences in CT across brain regions have been associated with individual differences in anxiety-related measures. For example, trait-anxiety has been negatively associated with CT in the right medial orbitofrontal cortex (mOFC; Kühn et al., 2011) and anxiety scores measured by the Hamilton Scale for Anxiety (HARS) were found to be associated with an increased CT of the anterior cingulate cortex (ACC; Donzuso et al., 2014). However, in another study (Blackmon et al., 2011), statistically significant associations between CT and trait-anxiety were found in left temporo-parietal regions. Thus, scattered findings across diverse brain regions have been reported regarding the association between $\mathrm{CT}$ and measures of anxiety.

\section{Rationale}

Evidence from studies on functional, biochemical, and structural neural data support the identification of biomarkers of risk for the development of anxiety disorders (Blackmon et al., 2011). Even though there has been a significant progress towards the development of the neural correlates of anxiety, to what extent changes in CT contribute to the development of anxiety disorders is still unclear. By conducting a scoping review aimed to scope the field of CT and its association with extant classic anxiety measures, we will have the opportunity to map the available literature, towards the identification of discrepancies and commonalities across studies.

\section{Objectives}

The main goal of this scoping review is to inform about the association between CT and anxiety-related measures. In specific, we aim to: (a) systematically identify and describe the studies about $\mathrm{CT}$ in what concerns magnetic resonance imaging (MRI) pre-processing and data analysis methods, and (b) summarize the main findings across anxiety disorders and healthy controls (HC).

Thus, the following research questions were formulated:

1. What is the state of the art concerning neuroimaging analysis and methods to measure and estimate CT?

2. What is known about the association between $\mathrm{CT}$ and measures of anxiety and fear?

3. What is known about the association between fear conditioning/fear extinction and CT?

4. What are the gaps in the current knowledge regarding the association between CT and anxiety-related measures?

\section{Methods}

The process of the scoping review was guided by the Arksey and O'Malley (2005) methodology framework of six stages, further developed by Levac et al. (2010) and the Joanna Briggs Institute (Peters et al., 2020). The research team found the methodology of the scoping 
review more appropriate because of the broad research questions formulated, aiming to perform a comprehensive scope of the existing research and literature.

In accordance with the optional consultation phase of the Arksey and O'Malley (2005) methodology, three experts on neuroimaging were consulted once and asked to provide insights beyond what is reported in the literature. Specifically, they provided input regarding: (a) the relevance of the association between CT and anxiety measures, (b) the key terms of the search, (c) authors of importance in the field, (d) the main gaps in the field, and (e) the main implications of the current study.

\section{Protocol}

The research questions, objectives, and methods (e.g., eligibility criteria) of the scoping review were chosen and stated a priori and described in a protocol (Sobral et al., 2020; Appendix A). This protocol was drafted using the guidelines and items of the Preferred Reporting Items for Systematic reviews and Meta-analyses extension for Scoping Reviews (PRISMA-ScR; Tricco et al., 2018).

\section{Eligibility Criteria}

To be included in the present review, the studies had to meet the following inclusion criteria:

(a) The study had to have CT as one of the outcome measures.

(b) The study focus had to be on the study of anxiety or fear, including fear conditioning and extinction. The following anxiety-related measures were considered: clinical selfreport measures, psychophysiological measures (skin conductance response [SCR] and heart rate variability), neurophysiological measures (fMRI), behavioral measures (avoidance), and measures of expectancy regarding fear response learning. Given the broader and multidisciplinary field, other measures of interest (e.g., genetics) were not included in the current review.

(c) The study had to evaluate the association between CT and anxiety-related measures.

(d) The study had to be conducted with healthy individuals or individuals diagnosed with at least one anxiety disorder (according to DSM-IV, DSM-IV-TR, DSM-5 and ICD10), aged between 18-60 years old. As anxiety and mood disorders often occur together, comorbidities between these two clinical diagnoses were included. OCD, acute stress disorder, and PTSD were excluded due to not being currently considered anxiety disorders. Aging was preliminarily excluded ( $>60$ years old), due to the expected and well-known negative correlations between age and CT in healthy aging (Salat et al., 2004), unless the studies in question controlled for age.

(e) The article had to be written in English or Portuguese. 
(f) The article had to be published between 2004-2020, given the technological progress of neuroimaging and the diffusion of CT methods and analysis after this year.

Papers were excluded if they did not meet the previous inclusion criteria or if the design of the study was qualitative, an opinion article or a review.

\section{Information Sources and Search Strategy}

A comprehensive literature search was conducted on the following electronic databases, from inception until January 17, 2020: PubMed, PsycINFO and PsycARTICLES (through Ovid), and Web of Science. In accordance with the methodology for scoping reviews from the Joanna Briggs Institute (Peters et al., 2020), a three-step strategy was used. The first search was conducted in the PubMed database, with the terms "(cortical thickness AND anx*) OR (cortical thickness AND fear)". After this initial search, keywords searched in all fields of the retrieved articles were analyzed, as well as the index terms. The next step included a second search across all databases, involving the identified key terms (Appendix B). Language (English and Portuguese) and time frame (2004-2020) limits were applied. In order to include all relevant papers, we also searched the reference lists of included articles. The final search strategy conducted in PsycINFO database can be found in Appendix C.

\section{Selection of Sources of Evidence}

The final search results were exported into the program Rayyan (Ouzzani et al., 2016), where one reviewer (MS) removed the duplicates across databases. Two reviewers (MS and SM) independently screened the title and abstracts of the retrieved articles, checking if they were eligible for the next step, a full-text retrieval. The selected full-text articles were analyzed by the same two reviewers, in terms of the eligibility criteria. Inter-rater disagreements were resolved through discussion until a consensus was reached. When the full consensus was not obtained, a third investigator (AG) provided her input. Cohen's kappa coefficient was used to calculate inter-rater agreement on study selection, based on the guidelines by Landis and Koch (1977): $k<.00$ as poor, $k \leq .20$ as slight, $k \leq .40$ as fair, $k \leq .60$ as moderate, $k \leq .80$ as substantial, and $k>0.81$ as almost perfect agreement.

\section{Data Charting Process}

The set of articles (17) was split into two (9 and 8) and assigned to a reviewer (MS and SM). The two reviewers independently extracted data from the assigned set of eligible articles and reviewed each other's extraction. After the comparison of each reviewer's charted data, disagreements were resolved through discussion between reviewers and with a third reviewer (AG) as well. A data-charting form was developed a priori by the authors (MS, SM and AG), based on common variables across articles. 


\section{Data Items}

The following domains were included in the data-extraction form: study, sample information (sample condition, sample size, mean age, sex, comorbidities and medication status, when applicable), methods (study design, setting, exclusion criteria, whole-brain or region-ofinterest [ROI], scanner strength, software used to process images, pre-processing methods, processing methods, quality check, statistical analysis, controlled variables, measures of anxiety and, when suited, fear procedure) and results (effect/no effect, direction of effect and brain regions implicated).

\section{Critical Appraisal of Individual Sources of Evidence}

Although this scoping review aims to map comprehensively all the available evidence, the research team sought to perform a critical appraisal of individual sources of evidence. The assessment of the methodological quality of the research was done using the Joanna Briggs Institute Critical Appraisal Checklist for Analytical Cross-Sectional Studies (Appendix D; Moola et al., 2020). This tool has been approved after an extensive peer review. Even though the checklist is mainly designed for use in systematic reviews, we thought it would be equally helpful for a scoping review. This checklist is comprised of eight questions, with four possible answers (yes, no, unclear or not applicable). It assesses the definition of exclusion criteria, the description of participants and setting, the measurement of the exposure and condition, the identification of cofounding factors and strategies to deal with these, the measurement of outcomes, and the statistical analysis employed. We adapted this checklist to the current scoping review, in the sense that exposure and condition pertain to $\mathrm{CT}$ and anxiety disorders, and the outcome concerns results according to anxiety measures. Results will be reported in terms of the overall appraisal (include, exclude, seek further info). However, this quality assessment will not be used to exclude studies in the present scoping review, but instead will be an additional source of information regarding the quality of the studies included.

\section{Synthesis of Results}

The articles were grouped according to the fear/anxiety response component assessed: selfreport measures, psychophysiological measures (e.g., SCR), and fMRI. The findings were synthesized through a tabular and narrative format. This was carried out by the primary author (MS) and reviewed by AG. A PRISMA flow diagram was also used to report the final results. 


\section{Results}

\section{Selection of Sources of Evidence}

After the removal of duplicates, 286 citations were reviewed. Of these, 243 were excluded based on the title and abstract. Of the remaining 43 full-text articles assessed for eligibility, 26 were excluded for different reasons: not assessing or reporting CT data (e.g., brain connectivity or volume only; $n=5$ ), wrong type of diagnosis/condition or excluded comorbidities (e.g., OCD; $n=$ 6), use of another measure of anxiety not included in the present review (e.g., genetic; $n=2$ ), wrong publication type (e.g., review; $n=1$ ), not assessing or reporting any measurement of anxiety (e.g., trauma or behavioral inhibition; $n=5$ ), anxiety not being the primary outcome (e.g., MDD was the primary focus; $n=3)$, and not assessing the association between anxiety measures and CT $(n=4)$. Subsequently, 17 articles were included in this review (Figure 1).

The inter-rater agreement for the selection of papers in the full-text phase was almost perfect $(k=.843, p<.001)$.

\section{Figure 1}

Flow-Chart of the Selection of Sources of Evidence

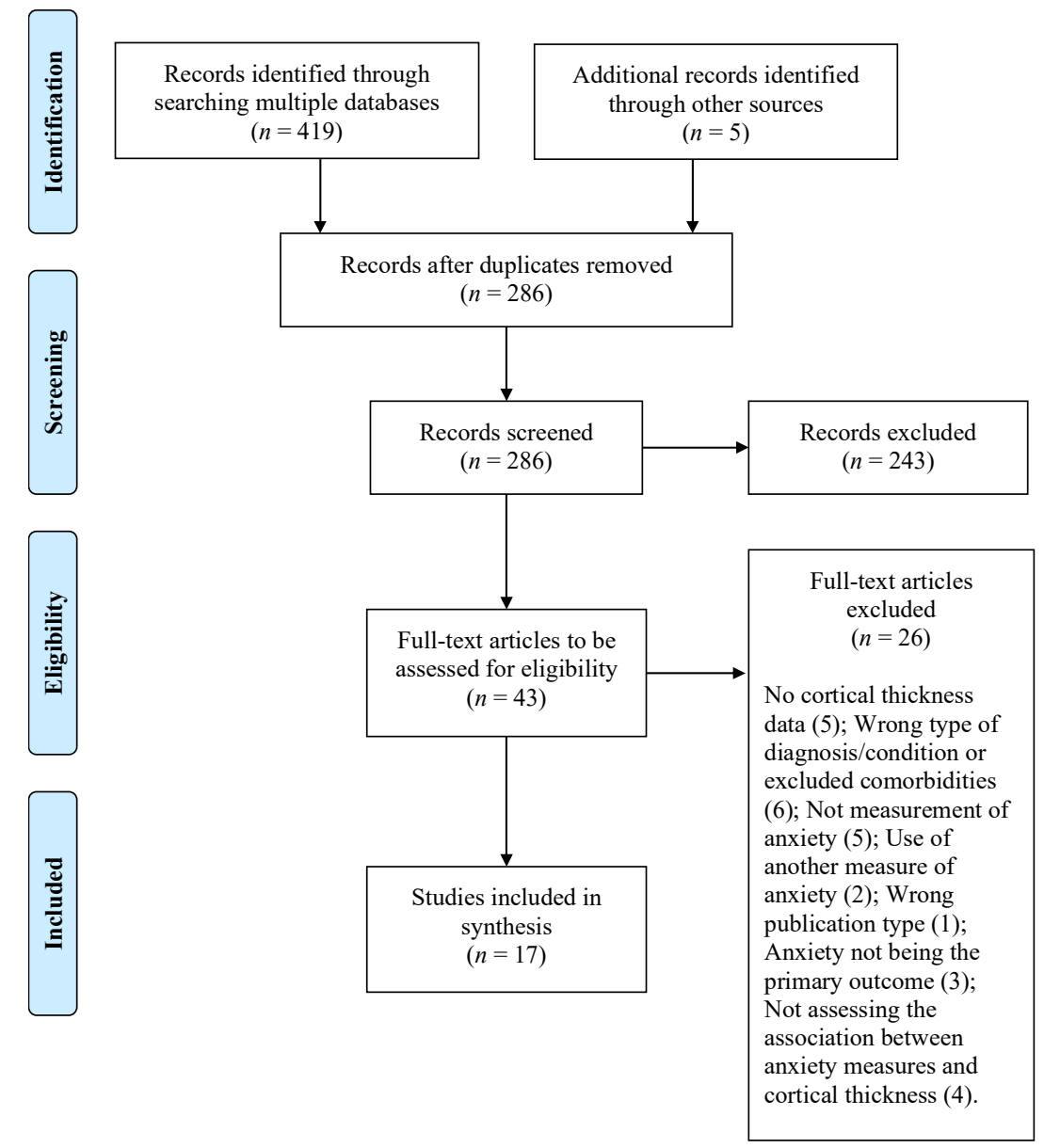




\section{Critical Appraisal Within Sources of Evidence}

The majority of studies $(n=15)$ can be considered of a high quality. However, three studies showed inadequate reporting, inadequate definition of exclusion criteria, and inadequate consideration of cofounding factors. Table 1 depicts the listed studies and the overall appraisal per study. The critical assessment step, including critical assessment items, can be found in detail in Appendix E.

\section{Table 1}

Critical Appraisal of Sources of Evidence Results

\begin{tabular}{lccc}
\hline \multicolumn{1}{c}{ Study } & \multicolumn{2}{c}{ Overall Appraisal } \\
\cline { 2 - 4 } & Include & Exclude & Seek Further Info \\
\hline Asami et al. (2018) & X & \\
Blackmon et al. (2011) & X & & \\
Brühl et al. (2014) & X & \\
Carnevali et al. (2019) & X & \\
Cha et al. (2014) & X & \\
Donzuso et al. (2014) & X & \\
Hartley et al. (2011) & & & \\
Kang et al. (2017) & X & \\
Kühn et al. (2011) & X & \\
Maggioni et al. (2019) & X & \\
Milad et al. (2005) & & \\
Milad et al. (2007) & & \\
Molent et al. (2018) & X & \\
Rosso et al. (2010) & X & \\
Syal et al. (2012) & X & \\
Zhao et al. (2017) & X & \\
Winkelmann et al. (2016) & X & \\
\hline
\end{tabular}

\section{Characteristics of Sources of Evidence and Synthesis of Results}

In order to better answer the scoping review questions, a summary of the literature is presented according to conceptual categories. The methodological characteristics of the included studies are presented in Table 2 and Table 3, while the main findings are reported in Table 4 and Table 5, according to the type of anxiety measure included (self-report or others). 


\section{Methodological Characteristics of the Included Studies}

Study Characteristics. As presented in Table 2, regarding study design and setting, all included studies ( $n=17$ ) were cross-sectional, quantitative, nonexperimental, and nonclinical ${ }^{2}$ (Asami et al., 2018; Blackmon et al., 2011; Brühl et al., 2014; Carnevali et al., 2019; Cha et al., 2014; Donzuso et al., 2014; Hartley et al., 2011; Kang et al., 2017; Kühn et al., 2011; Maggioni et al., 2019; Milad et al., 2005, 2007; Molent et al., 2018; Rosso et al., 2010; Syal et al., 2012; Winkelmann et al., 2016; Zhao et al., 2017). Of the 17 studies, 15 employed the FreeSurfer package software to process neuroimages (Asami et al., 2018; Blackmon et al., 2011; Brühl et al., 2014; Carnevali et al., 2019; Cha et al., 2014; Donzuso et al., 2014; Hartley et al., 2011; Kang et al., 2017; Kühn et al., 2011; Maggioni et al., 2019; Molent et al., 2018; Rosso et al., 2010; Syal et al., 2012; Winkelmann et al., 2016; Zhao et al., 2017), while two do not offer sufficient information for the clarification of this parameter (Milad et al., 2005, 2007).

The majority of studies reported the existence of exclusion criteria ( $n=14$; Asami et al., 2018; Blackmon et al., 2011; Brühl et al., 2014; Carnevali et al., 2019; Donzuso et al., 2014; Hartley et al., 2011; Kang et al., 2017; Kühn et al., 2011; Maggioni et al., 2019; Molent et al., 2018; Rosso et al., 2010; Syal et al., 2012; Winkelmann et al., 2016; Zhao et al., 2017).

Regarding scanner strength, 10 studies reported 3.0 T (Blackmon et al., 2011; Brühl et al., 2014; Cha et al., 2014; Hartley et al., 2011; Kang et al., 2017; Kühn et al., 2011; Molent et al., 2018; Rosso et al., 2010; Syal et al., 2012; Winkelmann et al., 2016 [study sample]; Zhao et al., 2017), while seven studies reported 1.5 T (Asami et al., 2018; Carnevali et al., 2019; Donzuso et al., 2014; Maggioni et al., 2019; Milad et al., 2005, 2007; Winkelmann et al., 2016 [replication sample]).

Four studies performed a whole-brain analysis (Asami et al., 2018; Blackmon et al., 2011; Syal et al., 2012; Zhao et al., 2017), while nine opted for a ROI analysis (Carnevali et al., 2019; Cha et al., 2014; Donzuso et al., 2014; Hartley et al., 2011; Kang et al., 2017; Maggioni et al., 2019; Milad et al., 2007; Molent et al., 2018; Rosso et al., 2010) and three integrated the two approaches (Brühl et al., 2014; Kühn et al., 2011; Milad et al., 2005). Also, one study employed a cluster-based analysis (Winkelmann et al., 2016).

Considering the ROI analysis, the following regions were included in the studies assessing self-report measures of anxiety $(n=12)$ : medial and lateral OFC $(n=4$; Carnevali et al., 2019; Donzuso et al., 2014; Maggioni et al., 2019; Molent et al., 2018), caudal and rostral ACC ( $n=3$;

\footnotetext{
${ }^{2}$ Cross-sectional refers to the concurrent evaluation of the variables (at one point in time). Quantitative methodology pertains to the way of testing theories through the analysis of the relationship between variables. Nonexperimental/correlational research corresponds to research without the manipulation of variables (Field, 2009). Nonclinical setting refers to studies that observe psychological/physiological basic processes but aren't conducted in a clinical setting.
} 
Carnevali et al., 2019; Donzuso et al., 2014; Molent et al., 2018), caudal and/or rostral middle frontal cortex ( $n=2$; Asami et al., 2018; Molent et al., 2018), posterior cingulate cortex $(n=2$; Carnevali et al., 2019; Molent et al., 2018), isthmus cingulate ( $n=2$; Carnevali et al., 2019; Molent et al., 2018), superior frontal ( $n=2$; Carnevali et al., 2019; Molent et al., 2018), insula ( $n=4$; Carnevali et al., 2019; Kang et al., 2017; Molent et al., 2018; Rosso et al., 2010), temporal pole ( $n$ =2; Kang et al., 2017; Molent et al., 2018), pars triangularis ( $n=1$; Kang et al., 2017), precentral gyrus ( $n=1$; Maggioni et al., 2019), fusiform gyrus ( $n=2$; Maggioni et al., 2019; Molent et al., 2018), postcentral gyrus ( $n=1$; Maggioni et al., 2019), entorhinal ( $n=1$; Molent et al., 2018), inferior and/or superior parietal cortex ( $n=2$; Maggioni et al., 2019; Molent et al., 2018), supra marginal ( $n=1$; Molent et al., 2018), and lateral occipital ( $n=1$; Molent et al., 2018).

Regarding the studies assessing other anxiety measures $(n=6)$, the main regions selected were as follows: ventromedial prefrontal cortex (vmPFC; $n=3$; Cha et al., 2014; Hartley et al., 2011; Winkelmann et al., 2016), dorsal ACC ( $n=2$; Hartley et al., 2011; Milad et al., 2007); rostral ACC $(n=2$; Carnevali et al., 2019; Milad et al., 2005), caudal ACC ( $n=1$; Carnevali et al., 2019), posterior cingulate cortex $(n=1$; Carnevali et al., 2019), isthmus cingulate gyrus $(n=1$; Carnevali et al., 2019), insular cortex ( $n=2$; Carnevali et al., 2019; Hartley et al., 2011), prefrontal cortex ( $n$ =2; Carnevali et al., 2019; Hartley et al., 2011), subcallosal cortex ( $n=1$; Milad et al., 2005), and $\operatorname{mOFC}(n=1$; Milad et al., 2005). 
Table 2

General Characteristics of the Included Studies $(n=17)$

\begin{tabular}{|c|c|c|c|c|c|}
\hline Study & Study design & Setting & Exclusion criteria & $\begin{array}{c}\text { Whole- } \\
\text { brain/ROI }\end{array}$ & $\begin{array}{l}\text { Scanner } \\
\text { strength }\end{array}$ \\
\hline $\begin{array}{l}\text { Asami et al. } \\
(2018)\end{array}$ & $\begin{array}{l}\text { Cross-sectional, } \\
\text { quantitative, } \\
\text { nonexperimental }\end{array}$ & Nonclinical & $\begin{array}{l}\text { Age }<19 \text { or }>57 \text { IQ }<75 \text {; left-handedness; history of epilepsy, } \\
\text { head trauma with loss of consciousness, neurological disorders, or } \\
\text { substance abuse; comorbid psychiatric disorders, other than MDD }\end{array}$ & Whole-brain & $1.5 \mathrm{~T}$ \\
\hline $\begin{array}{l}\text { Blackmon et } \\
\text { al. (2011) }\end{array}$ & $\begin{array}{l}\text { Cross-sectional, } \\
\text { quantitative, } \\
\text { nonexperimental }\end{array}$ & Nonclinical & $\begin{array}{l}\text { Prior history of depression, anxiety, psychosis, substance abuse, } \\
\text { psychotropic medication use, or neurological disorders; BAI score } \\
\text { greater than } 26 \text { or BDI-II score greater than } 29 \text {; left-handedness }\end{array}$ & Whole-brain & $3.0 \mathrm{~T}$ \\
\hline $\begin{array}{l}\text { Brühl et al. } \\
(2014)\end{array}$ & $\begin{array}{l}\text { Cross-sectional, } \\
\text { quantitative, } \\
\text { nonexperimental }\end{array}$ & Nonclinical & $\begin{array}{c}\text { Pregnancy; excessive consumption of alcohol, cigarettes, and } \\
\text { caffeine; contraindications against MRI; prior CBT; current and } \\
\text { previous mental and neurological disorders }\end{array}$ & Both & $3.0 \mathrm{~T}$ \\
\hline $\begin{array}{l}\text { Carnevali et al. } \\
(2019)\end{array}$ & $\begin{array}{l}\text { Cross-sectional, } \\
\text { quantitative, } \\
\text { nonexperimental }\end{array}$ & Nonclinical & $\begin{array}{c}\text { Age }<18 \text {; past head injury or neurological disorders; prior history } \\
\text { of major medical or psychiatric disorder (other than GAD); } \\
\text { cognitive impairment; history of substance or alcohol abuse or } \\
\text { dependence; diagnosis of heart disease; obesity; pregnancy, } \\
\text { claustrophobia or other general MRI exclusions }\end{array}$ & ROI & $1.5 \mathrm{~T}$ \\
\hline $\begin{array}{l}\text { Cha et al. } \\
(2014)\end{array}$ & $\begin{array}{l}\text { Cross-sectional, } \\
\text { quantitative, } \\
\text { nonexperimental }\end{array}$ & Nonclinical & Not available & ROI & $3.0 \mathrm{~T}$ \\
\hline $\begin{array}{l}\text { Donzuso et al. } \\
(2014)\end{array}$ & $\begin{array}{l}\text { Cross-sectional, } \\
\text { quantitative, } \\
\text { nonexperimental }\end{array}$ & Nonclinical & $\begin{array}{c}\text { Neurological or psychiatric disorders; substance abuse or other } \\
\text { medical problems; vascular brain lesions, brain tumor, and/or } \\
\text { marked cortical and/or subcortical atrophy on MRI scan; cognitive } \\
\text { impairment }\end{array}$ & ROI & $1.5 \mathrm{~T}$ \\
\hline
\end{tabular}




\begin{tabular}{|c|c|c|c|c|c|}
\hline Study & Study design & Setting & Exclusion criteria & $\begin{array}{l}\text { Whole- } \\
\text { brain/ROI }\end{array}$ & $\begin{array}{l}\text { Scanner } \\
\text { strength }\end{array}$ \\
\hline $\begin{array}{l}\text { Hartley et al. } \\
\text { (2011) }\end{array}$ & $\begin{array}{l}\text { Cross-sectional, } \\
\text { quantitative, } \\
\text { nonexperimental }\end{array}$ & Nonclinical & Absence of an initial SCR $(n=3)$ or failure to acquire a CR $(n=3)$ & ROI & $3.0 \mathrm{~T}$ \\
\hline $\begin{array}{l}\text { Kang et al. } \\
(2017)\end{array}$ & $\begin{array}{l}\text { Cross-sectional, } \\
\text { quantitative, } \\
\text { nonexperimental }\end{array}$ & Nonclinical & $\begin{array}{l}\text { Current diagnosis or lifetime history of schizophrenia, bipolar } \\
\text { disorder, anxiety disorders other than PD, alcohol and substance } \\
\text { abuse or dependence, mental retardation, serious medical or } \\
\text { neurological disorders, pregnancy, or any contraindications to MRI }\end{array}$ & ROI & $3.0 \mathrm{~T}$ \\
\hline $\begin{array}{l}\text { Kühn et al. } \\
(2011)\end{array}$ & $\begin{array}{c}\text { Cross-sectional, } \\
\text { quantitative, } \\
\text { nonexperimental }\end{array}$ & Nonclinical & $\begin{array}{c}\text { Medical, neurological and psychiatric disorders; subjects with a } \\
\text { family history (first degree) of axis I disorder; abnormalities in the } \\
\text { MRI, general medical disorders, or any clinically relevant } \\
\text { abnormalities }\end{array}$ & Both & $3.0 \mathrm{~T}$ \\
\hline $\begin{array}{l}\text { Maggioni et al. } \\
\text { (2019) }\end{array}$ & $\begin{array}{l}\text { Cross-sectional, } \\
\text { quantitative, } \\
\text { nonexperimental }\end{array}$ & Nonclinical & $\begin{array}{c}\text { Other comorbid axis I disorders, neurological or medical disorders } \\
\text { with possible effect on brain development; history of traumatic } \\
\text { head injury with loss of consciousness; and alcohol or substance } \\
\text { abuse }\end{array}$ & ROI & $1.5 \mathrm{~T}$ \\
\hline $\begin{array}{l}\text { Milad et al. } \\
\text { (2005) }\end{array}$ & $\begin{array}{l}\text { Cross-sectional, } \\
\text { quantitative, } \\
\text { nonexperimental }\end{array}$ & Nonclinical & Not available & Both & $1.5 \mathrm{~T}$ \\
\hline $\begin{array}{l}\text { Milad et al. } \\
\text { (2007) }\end{array}$ & $\begin{array}{l}\text { Cross-sectional, } \\
\text { quantitative, } \\
\text { nonexperimental }\end{array}$ & Nonclinical & Not available & ROI & $1.5 \mathrm{~T}$ \\
\hline $\begin{array}{l}\text { Molent et al. } \\
\text { (2018) }\end{array}$ & $\begin{array}{l}\text { Cross-sectional, } \\
\text { quantitative, } \\
\text { nonexperimental }\end{array}$ & Nonclinical & $\begin{array}{c}\text { History of traumatic head injury with loss of consciousness and } \\
\text { neurological or medical illness }\end{array}$ & ROI & $3.0 \mathrm{~T}$ \\
\hline $\begin{array}{l}\text { Rosso et al. } \\
(2010)\end{array}$ & $\begin{array}{c}\text { Cross-sectional, } \\
\text { quantitative, } \\
\text { nonexperimental }\end{array}$ & Nonclinical & $\begin{array}{c}\text { Current or past Axis I diagnoses, besides SAP; MRI } \\
\text { contraindications }\end{array}$ & ROI & $3.0 \mathrm{~T}$ \\
\hline
\end{tabular}




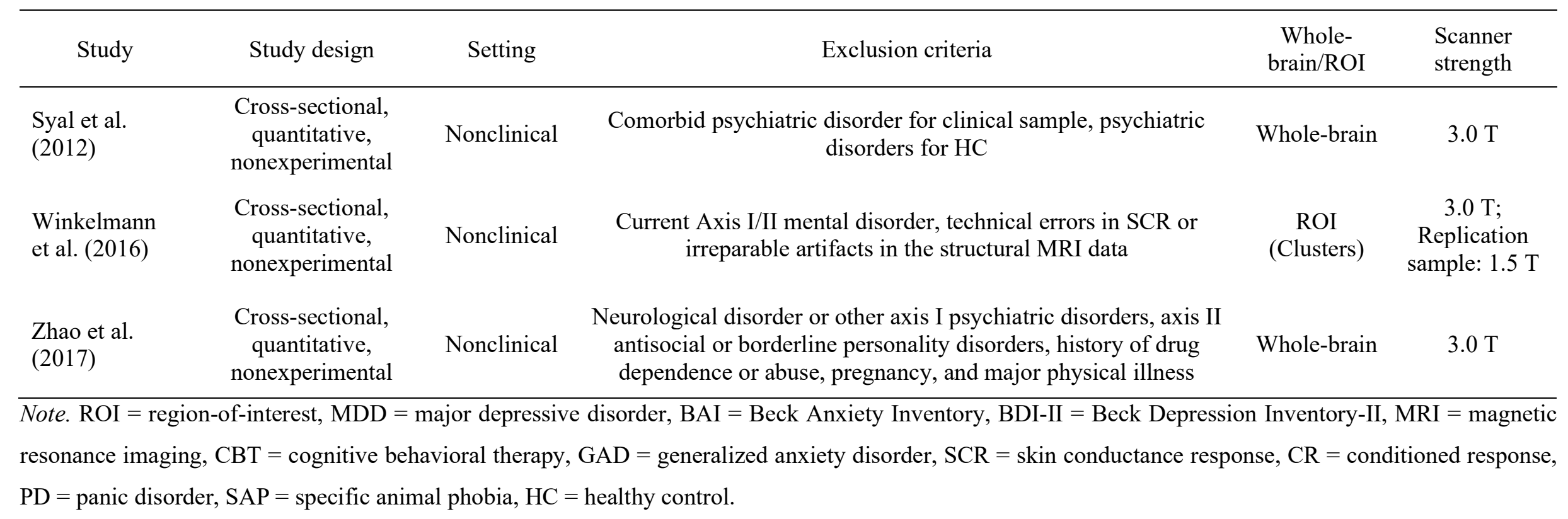


Image Processing Methods and Estimation of CT. As presented in Table 3, in terms of the methods used for measuring CT, almost all studies $(n=16)$ reported a surface-based approach (Asami et al., 2018; Blackmon et al., 2011; Brühl et al., 2014; Carnevali et al., 2019; Cha et al., 2014; Donzuso et al., 2014; Hartley et al., 2011; Kang et al., 2017; Kühn et al., 2011; Maggioni et al., 2019; Milad et al., 2005, 2007; Molent et al., 2018; Syal et al., 2012; Winkelmann et al., 2016; Zhao et al., 2017), consisting of, generally, image registration, intensity normalization, smoothing, brain extraction and segmentation, surface extraction, inflation or parameterization, and surface mapping (Fischl \& Dale, 2000). One study (Rosso et al., 2010) used the topological cortical parcellation (TCP) system, starting with volumetric segmentation data. The segmentation and parcellation of the cerebral cortex occurred through the Cardviews software and then CT differences were computed using FreeSurfer.

Studies differed in the Gaussian Kernel used, with the most commonly reported being 10 mm full-width at half-maximum (FWHM; $n=5$; Asami et al., 2018; Blackmon et al., 2011; Cha et al., 2014; Donzuso et al., 2014; Zhao et al., 2017), followed by 13 mm FWHM ( $n=2$; Milad et al., 2005, 2007), 30 mm FWHM ( $n=1$; Brühl et al., 2014), 5 mm FWHM ( $n=1$; Hartley et al., 2011), $20 \mathrm{~mm}$ FWHM ( $n=1$; Kühn et al., 2011) and $15 \mathrm{~mm}$ FWHM ( $n=1$; Winkelmann et al., 2016). Six studies did not report the gaussian kernel used (Carnevali et al., 2019; Kang et al., 2017; Maggioni et al., 2019; Molent et al., 2018; Rosso et al., 2010; Syal et al., 2012). Considering quality checking, 11 studies mention visually inspecting the cortical surfaces and manually correcting inaccuracies when necessary (Brühl et al., 2014; Hartley et al., 2011; Kang et al., 2017; Kühn et al., 2011; Maggioni et al., 2019; Milad et al., 2005, 2007; Molent et al., 2018; Syal et al., 2012; Winkelmann et al., 2016; Zhao et al., 2017). The remaining studies do not disclose this parameter ( $n=6$; Asami et al., 2018; Blackmon et al., 2011; Carnevali et al., 2019; Cha et al., 2014; Donzuso et al., 2014; Rosso et al., 2010). 
Table 3

MRI Processing Methods of the Included Studies $(n=17)$

\begin{tabular}{|c|c|c|c|c|}
\hline Study & Pre-processing & Processing & Quality check & $\begin{array}{l}\text { Software used to } \\
\text { process images }\end{array}$ \\
\hline $\begin{array}{l}\text { Asami et al. } \\
(2018)\end{array}$ & $\begin{array}{l}\text { FreeSurfer pre-processing package }{ }^{\mathrm{a}} \\
\text { The data were smoothed using a Gaussian kernel } \\
\text { with FWHM of } 10 \mathrm{~mm}\end{array}$ & $\begin{array}{l}\text { FreeSurfer } \\
\text { surface-based } \\
\text { analysis }^{\mathrm{b}}\end{array}$ & Not available & $\begin{array}{c}\text { FreeSurfer (version } \\
5.3 .0 \text { ) }\end{array}$ \\
\hline $\begin{array}{l}\text { Blackmon et al. } \\
\text { (2011) }\end{array}$ & $\begin{array}{l}\text { FreeSurfer pre-processing package }{ }^{\mathrm{a}} \\
\text { The data were smoothed using a Gaussian kernel } \\
\text { with FWHM of } 10 \mathrm{~mm}\end{array}$ & $\begin{array}{l}\text { FreeSurfer } \\
\text { surface-based } \\
\text { analysis }^{\mathrm{b}}\end{array}$ & Not available & $\begin{array}{c}\text { FreeSurfer (version } \\
5.0 .0 \text { ) }\end{array}$ \\
\hline $\begin{array}{l}\text { Brühl et al. } \\
\text { (2014) }\end{array}$ & $\begin{array}{l}\text { FreeSurfer pre-processing package } \\
\text { The data were smoothed using a Gaussian kernel } \\
\text { with a FWHM of } 30 \mathrm{~mm}\end{array}$ & $\begin{array}{l}\text { FreeSurfer } \\
\text { surface-based } \\
\text { analysis }^{\mathrm{b}}\end{array}$ & $\begin{array}{l}\text { Images were visually inspected for } \\
\text { inaccuracies and none of the } \\
\text { segmentations had to be excluded }\end{array}$ & $\begin{array}{c}\text { FreeSurfer (version } \\
4.5 .0 \text { ) }\end{array}$ \\
\hline $\begin{array}{l}\text { Carnevali et al. } \\
\text { (2019) }\end{array}$ & FreeSurfer pre-processing package ${ }^{a}$ & $\begin{array}{l}\text { FreeSurfer } \\
\text { surface-based } \\
\text { analysis }^{\mathrm{b}}\end{array}$ & Not available & $\begin{array}{l}\text { FreeSurfer (version } \\
\text { n.a.) }\end{array}$ \\
\hline Cha et al. (2014) & $\begin{array}{c}\text { FreeSurfer pre-processing package } \\
\text { For a vertex-wise analysis, the data were } \\
\text { smoothed with a surface-based Gaussian kernel } \\
\text { with FWHM of } 10 \mathrm{~mm}\end{array}$ & $\begin{array}{l}\text { FreeSurfer } \\
\text { surface-based } \\
\text { analysis }^{\mathrm{b}}\end{array}$ & Not available & $\begin{array}{l}\text { FreeSurfer (version } \\
\text { n.a.) }\end{array}$ \\
\hline $\begin{array}{l}\text { Donzuso et al. } \\
\text { (2014) }\end{array}$ & $\begin{array}{c}\text { FreeSurfer pre-processing package } \\
\text { Smoothing of data used a circularly symmetrical } \\
\text { Gaussian kernel with FWHM of } 10 \mathrm{~mm} \text {, with a } \\
\text { SD of } 10 \mathrm{~mm}\end{array}$ & $\begin{array}{l}\text { FreeSurfer } \\
\text { surface-based } \\
\text { analysis }^{\mathrm{b}}\end{array}$ & Not available & $\begin{array}{c}\text { FreeSurfer (version } \\
4.05 \text { ) }\end{array}$ \\
\hline $\begin{array}{l}\text { Hartley et al. } \\
\text { (2011) }\end{array}$ & $\begin{array}{l}\text { FreeSurfer pre-processing package }{ }^{\mathrm{a}} \\
\text { The data were smoothed with a surface-based } \\
\text { Gaussian kernel with FWHM of } 5 \mathrm{~mm}\end{array}$ & $\begin{array}{l}\text { FreeSurfer } \\
\text { surface-based } \\
\text { analysis }^{\mathrm{b}}\end{array}$ & $\begin{array}{l}\text { Images were visually inspected and } \\
\text { inaccuracies were manually corrected }\end{array}$ & $\begin{array}{l}\text { FreeSurfer (version } \\
\text { n.a.) }\end{array}$ \\
\hline
\end{tabular}




\begin{tabular}{|c|c|c|c|c|}
\hline Study & Pre-processing & Processing & Quality check & $\begin{array}{l}\text { Software used to } \\
\text { process images }\end{array}$ \\
\hline $\begin{array}{l}\text { Kang et al. } \\
(2017)\end{array}$ & FreeSurfer pre-processing package ${ }^{a}$ & $\begin{array}{l}\text { FreeSurfer } \\
\text { surface-based } \\
\text { analysis }\end{array}$ & $\begin{array}{l}\text { Images were visually inspected and } \\
\text { inaccuracies were manually corrected }\end{array}$ & $\begin{array}{c}\text { FreeSurfer (version } \\
5.3 .0 \text { ) }\end{array}$ \\
\hline $\begin{array}{l}\text { Kühn et al. } \\
\text { (2011) }\end{array}$ & $\begin{array}{l}\text { FreeSurfer pre-processing package } \\
\text { The data were smoothed with a surface-based } \\
\text { Gaussian kernel with FWHM of } 20 \mathrm{~mm}\end{array}$ & $\begin{array}{l}\text { FreeSurfer } \\
\text { surface-based } \\
\text { analysis }^{\mathrm{b}}\end{array}$ & $\begin{array}{l}\text { Images were visually inspected and } \\
\text { inaccuracies were manually corrected }\end{array}$ & $\begin{array}{l}\text { FreeSurfer (version } \\
\text { n.a.) }\end{array}$ \\
\hline $\begin{array}{l}\text { Maggioni et al. } \\
(2019)\end{array}$ & FreeSurfer pre-processing package ${ }^{a}$ & $\begin{array}{l}\text { FreeSurfer } \\
\text { surface-based } \\
\text { analysis }^{\mathrm{b}}\end{array}$ & $\begin{array}{l}\text { Images were visually inspected and } \\
\text { inaccuracies were manually corrected }\end{array}$ & $\begin{array}{c}\text { FreeSurfer (version } \\
5.3 .0 \text { ) }\end{array}$ \\
\hline $\begin{array}{l}\text { Milad et al. } \\
(2005)\end{array}$ & $\begin{array}{l}\text { FreeSurfer pre-processing package } \\
\text { The data were smoothed with a surface-based } \\
\text { Gaussian kernel with FWHM of } \approx 13 \mathrm{~mm}\end{array}$ & $\begin{array}{l}\text { FreeSurfer } \\
\text { surface-based } \\
\text { analysis }\end{array}$ & $\begin{array}{l}\text { Images were visually inspected, and } \\
\text { inaccuracies were manually corrected }\end{array}$ & $\begin{array}{l}\text { Insufficient } \\
\text { information }\end{array}$ \\
\hline $\begin{array}{l}\text { Milad et al. } \\
(2007)^{c}\end{array}$ & see Milad et al. (2005) & $\begin{array}{l}\text { see Milad et al. } \\
\text { (2005) }\end{array}$ & See Milad et al. (2005) & $\begin{array}{l}\text { Insufficient } \\
\text { information }\end{array}$ \\
\hline $\begin{array}{l}\text { Molent et al. } \\
(2018)\end{array}$ & FreeSurfer pre-processing package ${ }^{a}$ & $\begin{array}{l}\text { FreeSurfer } \\
\text { surface-based } \\
\text { analysis }^{\mathrm{b}}\end{array}$ & $\begin{array}{l}\text { Images were visually inspected and } \\
\text { inaccuracies were manually corrected }\end{array}$ & $\begin{array}{c}\text { FreeSurfer (version } \\
5.3 .0 \text { ) }\end{array}$ \\
\hline $\begin{array}{l}\text { Rosso et al. } \\
(2010)\end{array}$ & TCP system $^{d}$ & $\begin{array}{l}\text { Cardviews and } \\
\text { FreeSurfer } \\
\text { analysis }\end{array}$ & Not available & $\begin{array}{l}\text { Cardviews and } \\
\text { FreeSurfer (version } \\
\text { n.a.) }\end{array}$ \\
\hline $\begin{array}{l}\text { Syal et al. } \\
(2012)\end{array}$ & FreeSurfer pre-processing package ${ }^{a}$ & $\begin{array}{l}\text { FreeSurfer } \\
\text { surface-based } \\
\text { analysis }\end{array}$ & $\begin{array}{l}\text { Images were visually inspected and } \\
\text { inaccuracies were manually corrected }\end{array}$ & $\begin{array}{c}\text { FreeSurfer (version } \\
4.5 \text { ) }\end{array}$ \\
\hline \multicolumn{3}{|c|}{ Association Between Cortical Thickness and Anxiety Measures: A Scoping Review } & 22 & \\
\hline
\end{tabular}




\begin{tabular}{|c|c|c|c|c|}
\hline Study & Pre-processing & Processing & Quality check & $\begin{array}{l}\text { Software used to } \\
\text { process images }\end{array}$ \\
\hline $\begin{array}{l}\text { Winkelmann et } \\
\text { al. (2016) }\end{array}$ & $\begin{array}{l}\text { FreeSurfer pre-processing package } \\
\text { The data were smoothed with a surface-based } \\
\text { Gaussian kernel with FWHM of } 15 \mathrm{~mm}\end{array}$ & $\begin{array}{l}\text { FreeSurfer } \\
\text { surface-based } \\
\text { analysis }^{\mathrm{b}}\end{array}$ & $\begin{array}{l}\text { Images were visually inspected and } \\
\text { inaccuracies were manually corrected }\end{array}$ & $\begin{array}{c}\text { FreeSurfer (version } \\
5.0 \text { ) }\end{array}$ \\
\hline $\begin{array}{l}\text { Zhao et al. } \\
(2017)\end{array}$ & $\begin{array}{l}\text { FreeSurfer pre-processing package } \\
\text { The data were smoothed with a surface-based } \\
\text { Gaussian kernel with FWHM of } 10 \mathrm{~mm}\end{array}$ & $\begin{array}{l}\text { FreeSurfer } \\
\text { surface-based } \\
\text { analysis }\end{array}$ & $\begin{array}{l}\text { Segmentation results were visually } \\
\text { inspected and manually edited by adding } \\
\text { control points, if necessary }\end{array}$ & $\begin{array}{c}\text { FreeSurfer (version } \\
5.1 .0)\end{array}$ \\
\hline
\end{tabular}

Note. FWHM = full-width at half-maximum, n.a. $=$ not available, TCP = topological cortical parcellation.

${ }^{\text {a }}$ The FreeSurfer pre-processing package includes nonuniformity correction and registration to stereotaxic space, removal of intensity inhomogeneity artifacts, and optionally normalized intensities.

${ }^{\mathrm{b}}$ The FreeSurfer surface-based analysis consists of five steps: (1) masking the brain and tissue classification into cortical gray matter, (2) surface fitting (fitting two polyhedral meshes to the inside [white/gray] and outside [pial, gray/cerebrospinal fluid] boundaries of the cortex), (3) thickness measurements (measuring CT as the distance between the grey matter and the grey/white matter boundary surfaces), (4) surface-based smoothing, and (5) surface-based alignment of the thickness maps (Wagstyl \& Lerch, 2018). For more details see Fischl and Dale (2000).

${ }^{c}$ This study analyzed data collected in Milad et al. (2005).

${ }^{\mathrm{d}}$ MRI intensities were normalized. The cerebral cortex was segmented and parcellated using the Cardviews software, following a manual procedure, while cortical surface analysis was computed using FreeSurfer. The surface was tessellated, smoothed, and inflated, and the cortical parcellation map was overlayed on the inflated surface. An intensity gradient was created as a function of the distance from the white matter surface. The exterior surface was generated by being pushed outward from the white matter surface and thickness maps were then created across the cerebral cortex. A FreeSurfer algorithm was used to create the "pial" surface, while the white matter surface was transferred to spherical coordinates and registered to the average Montreal Neurological Institute (MNI) brain. For more details see Makris et al. (2006). 


\section{CT and Measures of Anxiety}

The most frequently used measures of anxiety in the included studies were self-report anxiety-related symptoms/processes ( $n=12$; Asami et al., 2018; Blackmon et al., 2011; Brühl et al., 2014; Carnevali et al., 2019; Donzuso et al., 2014; Kang et al., 2017; Kühn et al., 2011; Maggioni et al., 2019; Molent et al., 2018; Rosso et al., 2010; Syal et al., 2012; Zhao et al., 2017), followed by psychophysiological ( $n=5$; Carnevali et al., 2019; Hartley et al., 2011; Milad et al., 2005, 2007; Winkelmann et al., 2016), and fMRI ( $n=1$; Cha et al., 2014).

Self-Report Measures of Anxiety Symptoms. Regarding self-report measures of anxiety related-symptoms or processes, the following were reported (Table 4): STAI or STAI-trait scale ( $n$ = 5; Blackmon et al., 2011; Brühl et al., 2014; Carnevali et al., 2019; Donzuso et al., 2014; Kühn et al., 2011), HARS ( $n=3$; Donzuso et al., 2014; Maggioni et al., 2019; Molent et al., 2018), BAI ( $n=2$; Blackmon et al., 2011; Kang et al., 2017), Anxiety Sensitivity Index-Revised (ASI-R; $n=$ 1; Kang et al., 2017), and the 16-item Anxiety Sensitivity Index (ASI; $n=1$; Rosso et al., 2010). Additionally, the following measures to assess clinical/symptom severity were reported: Penn State Worry Questionnaire (PSWQ; $n=1$; Carnevali et al., 2019), Panic Disorder Severity Scale (PDSS; $n=2$; Asami et al., 2018; Kang et al., 2017), Liebowitz Social Anxiety Scale (LSAS; $n=3$; Brühl et al., 2014; Syal et al., 2012; Zhao et al., 2017), Social Phobia Scale (SPS; $n=1$; Brühl et al., 2014), and Social Interaction Anxiety Scale (SIAS; $n=1$; Brühl et al., 2014).

In terms of the analytical strategy selected, the most common analyses undertaken were correlations ( $n$ =9; Asami et al., 2018; Brühl et al., 2014; Carnevali et al., 2019; Kang et al., 2017; Maggioni et al., 2019; Molent et al., 2018; Rosso et al., 2010; Syal et al., 2012; Zhao et al., 2017), namely Pearson's ( $n=5$; Kang et al., 2017; Maggioni et al., 2019; Molent et al., 2018; Rosso et al., 2010; Syal et al., 2012) or Spearman's correlations ( $n=1$; Asami et al., 2018). Of these, five studies reported the use of partial correlations (Carnevali et al., 2019; Maggioni et al., 2019; Molent et al., 2018; Syal et al., 2012; Zhao et al., 2017), while one used bivariate correlations (Brühl et al., 2014). The General Linear Model (GLM) was also used ( $n=2$; Blackmon et al., 2011; Rosso et al., 2010), as well as the multiple regression ( $n=1$; Donzuso et al., 2014), post hoc stepwise linear regressions ( $n=1$; Kühn et al., 2011) and the $t$-statistics with regression approach ( $n=1$; Kühn et al., 2011).

Nine studies included samples of patients with anxiety disorders (Asami et al., 2018; Brühl et al., 2014; Carnevali et al., 2019; Kang et al., 2017; Maggioni et al., 2019; Molent et al., 2018; Rosso et al., 2010; Syal et al., 2012; Zhao et al., 2017), namely PD ( $n=3$; Asami et al., 2018; Kang et al., 2017; Maggioni et al., 2019), SAD ( $n=3$; Brühl et al., 2014; Syal et al., 2012; Zhao et al., 2017), GAD ( $n=3$; Carnevali et al., 2019; Maggioni et al., 2019; Molent et al., 2018) and SP ( $n=$ 1; Rosso et al., 2010). Studies observing samples of patients with anxiety disorders compared their data with a group of HC. Three studies observed only healthy individuals (Blackmon et al., 2011; 
Donzuso et al., 2014; Kühn et al., 2011). Regarding comorbidities, the most frequently reported was current or past history of MDD or depressive episode ( $n=3$; Asami et al., 2018; Brühl et al., 2014; Molent et al., 2018), followed by personality disorders ( $n=1$; Maggioni et al., 2019). Two studies reported comorbid anxiety disorders, namely PD and agoraphobia ( $n=1$; Kang et al., 2017) and GAD and SP ( $n=1$; Molent et al., 2018).

Six studies observing clinical samples state the use of medication ( $n=6$; Asami et al., 2018; Brühl et al., 2014; Kang et al., 2017; Maggioni et al., 2019; Molent et al., 2018; Syal et al., 2012), namely antidepressant (e.g., selective serotonin reuptake inhibitors; $n=5$; Asami et al., 2018; Brühl et al., 2014; Kang et al., 2017; Maggioni et al., 2019; Molent et al., 2018), benzodiazepines ( $n=3$; Asami et al., 2018; Kang et al., 2017; Molent et al., 2018), and antipsychotic ( $n=1$; Molent et al., 2018). One study reported one patient who used benzodiazepines intermittently (Syal et al., 2012). Three of the clinical samples were medication free (Carnevali et al., 2019; Rosso et al., 2010; Zhao et al., 2017).

Across the included studies, the most commonly controlled variables were age $(n=9$; Blackmon et al., 2011; Carnevali et al., 2019; Donzuso et al., 2014; Kühn et al., 2011; Maggioni et al., 2019; Molent et al., 2018; Rosso et al., 2010; Syal et al., 2012; Zhao et al., 2017) and sex ( $n=$ 6; Donzuso et al., 2014; Kühn et al., 2011; Maggioni et al., 2019; Molent et al., 2018; Rosso et al., 2010; Zhao et al., 2017), followed by average CT ( $n=2$; Carnevali et al., 2019; Rosso et al., 2010), diagnosis ( $n=1$; Maggioni et al., 2019), scan site ( $n=1$; Rosso et al., 2010) and intracranial volume ( $n=1$; Syal et al., 2012). Three studies did not disclose the existence of controlled variables for the analysis of the association between $\mathrm{CT}$ and anxiety measures, although variables such as age were controlled in previous analysis (e.g., analysis of covariance; Asami et al., 2018; Brühl et al., 2014; Kang et al., 2017).

Regarding the findings about the association between CT and STAI, in what concerns studies observing clinical samples, one study with SAD patients did not find a statistically significant effect between these variables (Brühl et al., 2014). However, a second study that assessed GAD patients (Carnevali et al., 2019) found a statistically significant negative association between STAI and the CT in the left mOFC and right isthmus cingulate gyrus, both in GAD and HC. Amongst the studies comprising only healthy individuals, one did not find statistically significant effects (Donzuso et al., 2014), while two others did find a significant association between STAI-trait scale and CT (Blackmon et al., 2011; Kühn et al., 2011). Blackmon et al. (2011) found a positive association between anxiety scores and the $\mathrm{CT}$ in the left temporo-parietal region, spanning the posterior portion of the superior temporal sulcus and the inferior parietal region. Kühn et al. (2011) found a negative association between anxiety scores and the CT in the right mOFC (right gyrus rectus). 
Considering HARS, in clinical studies, focusing on PD patients $(n=1$; Maggioni et al., 2019) and GAD patients ( $n=2$; Maggioni et al., 2019; Molent et al., 2018), statistically significant effects were not found. However, although not statistically significant, a tendency for a positive association was found between HARS scores and CT in the left middle temporal cortex in one study with GAD patients (Molent et al., 2018). In terms of healthy population, one study (Donzuso et al., 2014) found a statistically significant positive association between HARS and the CT of the caudal and rostral ACC. Also, the authors found that HARS scores were significantly predicted by the anatomical variance (Donzuso et al., 2014).

With regard to BAI, a study focusing on PD (Kang et al., 2017) found a statistically significant positive association between $\mathrm{CT}$ and $\mathrm{BAI}$ scores in the left parts triangularis. In a sample of healthy individuals, one study (Blackmon et al., 2011) found a statistically significant positive association for this instrument in the lateral $\mathrm{OFC}$ and temporo-parietal regions, with the latter spanning the posterior portion of the superior temporal sulcus and the inferior parietal region.

Concerning ASI-R, one study (Kang et al., 2017; PD) found a statistically significant positive association between CT and ASI-R scores in the left pars triangularis, as well as a negative association in the right temporal pole. For ASI, the study by Rosso et significant effects in both the GLM and correlations analysis. First, the authors found a significant interaction between group and ASI scores, with higher ASI scores predicting increased CT in the right anterior insula, in the SAP group. Second, there was a positive association between ASI scores and CT in the right anterior insula in SAP subjects, but not in HC (Rosso et al., 2010).

With relation to PSWQ, one study (Carnevali et al., 2019) found a statistically significant negative association between PSWQ and CT in the left mOFC and right isthmus cingulate gyrus, both in the GAD group and in HC.

Considering PDSS, a statistically significant negative association between PDSS and CT was found in the left rostral middle frontal cortex (Asami et al., 2018), as well as a statistically significant positive association in the left pars triangularis (Kang et al., 2017), in PD patients

In terms of the LSAS instrument, two studies found statistically significant effects, namely a positive association between LSAS and CT in the right anterior insula (Brühl et al., 2014) and a negative association in the left postcentral cortex (Syal et al., 2012), in SAD patients. Although not statistically significant, a negative association was found between LSAS and CT in the right postcentral cortex (Syal et al., 2012). One study (Zhao et al., 2017) did not find statistically significant effects. For SPS, Brühl et al. (2014) found a statistically significant positive association between SPS scores and CT in the right anterior insula and parahippocampal gyrus in SAD patients. Finally, one study did not find statistically significant effects regarding the association between SIAS and CT (Brühl et al., 2014). 


\section{Table 4}

Association Between CT and Self-Report Measures of Anxiety $(n=12)$

\begin{tabular}{|c|c|c|c|c|c|c|c|c|c|}
\hline \multirow[b]{2}{*}{ Study ${ }^{\mathrm{a}}$} & \multirow[b]{2}{*}{$\begin{array}{l}\text { Measures of } \\
\text { anxiety }\end{array}$} & \multicolumn{5}{|c|}{ Sample information } & \multicolumn{3}{|c|}{ Main findings } \\
\hline & & Group $(n)$ & Comorbidity & $\begin{array}{l}\text { Medication } \\
\text { status }\end{array}$ & $\begin{array}{l}\text { Mean } \\
\text { age } \\
\text { (SD) }\end{array}$ & $\begin{array}{l}\text { Sex } \\
(\mathrm{M} / \mathrm{F})\end{array}$ & $\begin{array}{l}\text { Significant } \\
\text { effect }\end{array}$ & $\begin{array}{l}\text { Direction of } \\
\text { effect }\end{array}$ & Brain regions \\
\hline \multirow{2}{*}{$\begin{array}{l}\text { Asami et } \\
\text { al. (2018) }\end{array}$} & \multirow{2}{*}{ PDSS } & $\begin{array}{c}\mathrm{PD} \\
(n=38)\end{array}$ & $\begin{array}{l}\text { Past history } \\
\text { of MDD } \\
(n=6)\end{array}$ & Medicated & $\begin{array}{c}38.8 \\
(10.1)\end{array}$ & $13 / 25$ & \multirow{2}{*}{ Yes } & \multirow{2}{*}{$\begin{array}{c}\text { Negative } \\
\text { association (PD } \\
\text { group) }\end{array}$} & \multirow{2}{*}{$\begin{array}{l}\text { Left rostral middle } \\
\text { frontal cortex }\end{array}$} \\
\hline & & $\begin{array}{c}\mathrm{HC} \\
(n=38)\end{array}$ & $\begin{array}{c}\text { Not } \\
\text { applicable }\end{array}$ & Not applicable & $\begin{array}{c}37.8 \\
(10.3)\end{array}$ & $13 / 25$ & & & \\
\hline \multirow{2}{*}{$\begin{array}{l}\text { Blackmon } \\
\text { et al. } \\
\text { (2011) }\end{array}$} & \multirow{2}{*}{$\begin{array}{l}\text { BAI and } \\
\text { STAI-trait } \\
\text { scale }\end{array}$} & $\begin{array}{c}\text { BAI } \\
(n=25)\end{array}$ & $\begin{array}{l}\text { Healthy } \\
\text { sample }\end{array}$ & Not applicable & $40(12)$ & $13 / 12$ & Yes for BAI & $\begin{array}{l}\text { Positive } \\
\text { association }\end{array}$ & $\begin{array}{l}\text { 1OFC and temporo- } \\
\text { parietal regions }\end{array}$ \\
\hline & & $\begin{array}{l}\text { STAI-trait } \\
(n=18)\end{array}$ & $\begin{array}{l}\text { Healthy } \\
\text { sample }\end{array}$ & Not applicable & $39(11)$ & $10 / 8$ & $\begin{array}{l}\text { Yes for STAI- } \\
\text { trait }\end{array}$ & $\begin{array}{l}\text { Positive } \\
\text { association }\end{array}$ & $\begin{array}{l}\text { Left temporo- } \\
\text { parietal regions }\end{array}$ \\
\hline \multirow{2}{*}{$\begin{array}{l}\text { Brühl et al. } \\
\text { (2014) }\end{array}$} & \multirow{2}{*}{$\begin{array}{l}\text { STAI-trait } \\
\text { scale, LSAS, } \\
\text { SPS, and } \\
\text { SIAS }\end{array}$} & $\begin{array}{c}\text { SAD } \\
(n=46)\end{array}$ & $\begin{array}{l}\text { Current } \\
\text { depressive } \\
\text { episode } \\
(n=1)\end{array}$ & $\begin{array}{l}\text { Medicated, } \\
\text { stable for at } \\
\text { least } 4 \text { weeks }\end{array}$ & $\begin{array}{c}33.13 \\
(10.61)\end{array}$ & $29 / 17$ & $\begin{array}{l}\text { Yes for the ROI } \\
\text { analysis and } \\
\text { LSAS }\end{array}$ & $\begin{array}{c}\text { Positive } \\
\text { association } \\
\text { (SAD group) }\end{array}$ & Right anterior insula \\
\hline & & $\begin{array}{c}\mathrm{HC} \\
(n=46)\end{array}$ & $\begin{array}{c}\text { Not } \\
\text { applicable }\end{array}$ & Not applicable & $\begin{array}{l}32.96 \\
(8.87)\end{array}$ & $29 / 17$ & $\begin{array}{l}\text { Yes for the ROI } \\
\text { analysis and } \\
\text { SPS }\end{array}$ & $\begin{array}{c}\text { Positive } \\
\text { association } \\
\text { (SAD group) }\end{array}$ & $\begin{array}{c}\text { Right anterior insula } \\
\text { and } \\
\text { parahippocampal } \\
\text { gyrus }\end{array}$ \\
\hline
\end{tabular}




\begin{tabular}{|c|c|c|c|c|c|c|c|c|c|}
\hline \multirow[b]{2}{*}{ Study ${ }^{\mathrm{a}}$} & \multirow[b]{2}{*}{$\begin{array}{l}\text { Measures of } \\
\text { anxiety }\end{array}$} & \multicolumn{5}{|c|}{ Sample information } & \multicolumn{3}{|c|}{ Main findings } \\
\hline & & Group $(n)$ & Comorbidity & $\begin{array}{l}\text { Medication } \\
\text { status }\end{array}$ & $\begin{array}{l}\text { Mean } \\
\text { age } \\
\text { (SD) }\end{array}$ & $\begin{array}{l}\text { Sex } \\
(\mathrm{M} / \mathrm{F})\end{array}$ & $\begin{array}{l}\text { Significant } \\
\text { effect }\end{array}$ & $\begin{array}{l}\text { Direction of } \\
\text { effect }\end{array}$ & Brain regions \\
\hline $\begin{array}{l}\text { Carnevali } \\
\text { et al. } \\
\text { (2019) }\end{array}$ & $\begin{array}{l}\text { STAI-trait } \\
\text { and PSWQ }\end{array}$ & $\begin{array}{c}\text { GAD } \\
(n=17)\end{array}$ & None & $\begin{array}{l}\text { Medication } \\
\text { free }\end{array}$ & $\begin{array}{l}30.7 \\
(2.0)\end{array}$ & $\begin{array}{c}\text { All } \\
\text { female }\end{array}$ & $\begin{array}{l}\text { Yes for both } \\
\text { STAI-trait and } \\
\text { PSWQ }\end{array}$ & $\begin{array}{l}\text { Negative } \\
\text { association in } \\
\text { both groups }\end{array}$ & $\begin{array}{l}\text { Left mOFC and } \\
\text { right isthmus } \\
\text { cingulate gyrus }\end{array}$ \\
\hline $\begin{array}{l}\text { Donzuso et } \\
\text { al. (2014) }\end{array}$ & $\begin{array}{l}\text { STAI and } \\
\text { HARS }\end{array}$ & $\begin{array}{l}\text { Healthy } \\
(n=121)\end{array}$ & $\begin{array}{c}\text { Not } \\
\text { applicable }\end{array}$ & Not applicable & $\begin{array}{c}38.7 \\
(15.1)\end{array}$ & $54 / 67$ & $\begin{array}{l}\text { Yes for HARS, } \\
\text { but not for } \\
\text { STAI }\end{array}$ & $\begin{array}{l}\text { Positive } \\
\text { association }\end{array}$ & $\begin{array}{c}\text { Caudal and rostral } \\
\text { ACC }\end{array}$ \\
\hline \multirow[t]{2}{*}{$\begin{array}{l}\text { Kang et al. } \\
\text { (2017) }\end{array}$} & \multirow[t]{2}{*}{$\begin{array}{l}\text { PDSS, ASI- } \\
\text { R, and BAI }\end{array}$} & & & & & & & & \\
\hline & & $\begin{array}{c}\mathrm{HC} \\
(n=30)\end{array}$ & $\begin{array}{c}\text { Not } \\
\text { applicable }\end{array}$ & Not applicable & $\begin{array}{l}34.87 \\
(8.80)\end{array}$ & $16 / 14$ & $\begin{array}{l}\text { Yes, between } \\
\text { CT and ASI-R } \\
\text { score }\end{array}$ & $\begin{array}{l}\text { Negative } \\
\text { association } \\
\text { (PD group) }\end{array}$ & Right temporal pole \\
\hline
\end{tabular}




\begin{tabular}{|c|c|c|c|c|c|c|c|c|c|}
\hline \multirow[b]{2}{*}{ Study ${ }^{\mathrm{a}}$} & \multirow[b]{2}{*}{$\begin{array}{l}\text { Measures of } \\
\text { anxiety }\end{array}$} & \multicolumn{5}{|c|}{ Sample information } & \multicolumn{3}{|c|}{ Main findings } \\
\hline & & Group $(n)$ & Comorbidity & $\begin{array}{l}\text { Medication } \\
\text { status }\end{array}$ & $\begin{array}{l}\text { Mean } \\
\text { age } \\
\text { (SD) }\end{array}$ & $\begin{array}{l}\text { Sex } \\
(\mathrm{M} / \mathrm{F})\end{array}$ & $\begin{array}{l}\text { Significant } \\
\text { effect }\end{array}$ & $\begin{array}{l}\text { Direction of } \\
\text { effect }\end{array}$ & Brain regions \\
\hline $\begin{array}{l}\text { Kühn et al. } \\
\text { (2011) }\end{array}$ & STAI-trait & $\begin{array}{l}\text { Healthy } \\
(n=34)\end{array}$ & $\begin{array}{c}\text { Not } \\
\text { applicable }\end{array}$ & Not applicable & $30.5(?)$ & $14 / 20$ & Yes & $\begin{array}{l}\text { Negative } \\
\text { association }\end{array}$ & $\begin{array}{l}\text { Right mOFC (right } \\
\text { gyrus rectus) }\end{array}$ \\
\hline $\begin{array}{l}\text { Maggioni } \\
\text { et al. } \\
\text { (2019) }\end{array}$ & HARS & $\begin{array}{c}\text { PD } \\
(n=11)\end{array}$ & $\begin{array}{l}\text { Personality } \\
\text { disorders } \\
(n=3)\end{array}$ & Medicated & $\begin{array}{l}33.55 \\
(9.53)\end{array}$ & $5 / 6$ & No & Not applicable & Not applicable \\
\hline \multirow[t]{2}{*}{$\begin{array}{l}\text { Molent et } \\
\text { al. (2018) }\end{array}$} & HARS & $\begin{array}{c}\text { GAD } \\
(n=31)\end{array}$ & $\begin{array}{c}\text { MDD } \\
(n=1), \mathrm{SP} \\
(n=1), \\
\text { SP and past } \\
\text { MDD } \\
(n=1)\end{array}$ & Medicated & $\begin{array}{c}43.77 \\
(14.61)\end{array}$ & $11 / 20$ & \multirow[t]{2}{*}{$\mathrm{No}^{\mathrm{b}}$} & \multirow[t]{2}{*}{ Not applicable } & \multirow[t]{2}{*}{ Not applicable } \\
\hline & & $\begin{array}{c}\mathrm{HC} \\
(n=31)\end{array}$ & $\begin{array}{c}\text { Not } \\
\text { applicable }\end{array}$ & Not applicable & $\begin{array}{c}39.68 \\
(13.48)\end{array}$ & $14 / 17$ & & & \\
\hline
\end{tabular}




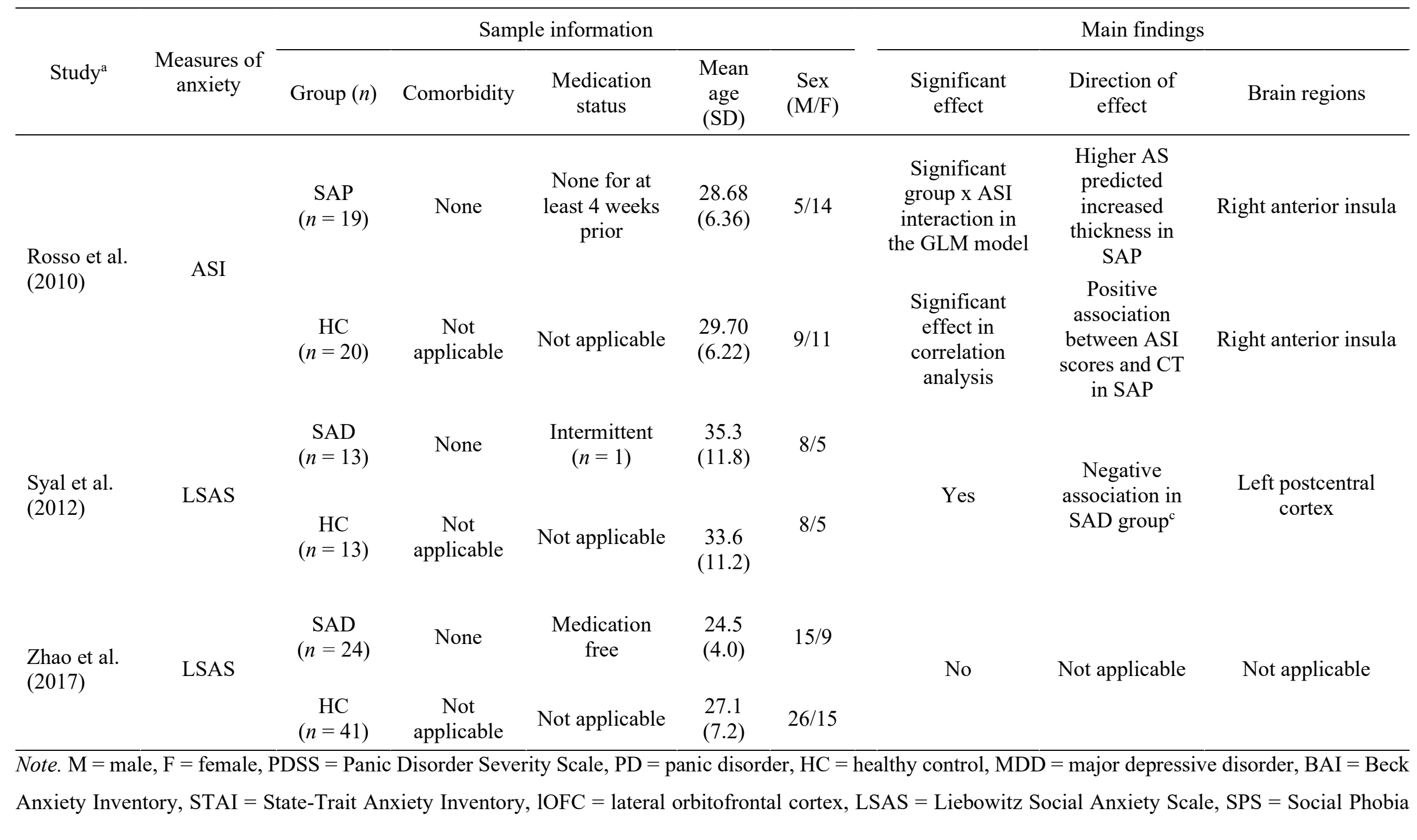


Scale, SIAS = Social Interaction Anxiety Scale, SAD = social anxiety disorder, ROI = region-of-interest, PSWQ = Penn State Worry Questionnaire, GAD = generalized anxiety disorder, $\mathrm{mOFC}=$ medial orbitofrontal cortex, HARS $=$ Hamilton Scale for Anxiety, ACC $=$ anterior cingulate cortex, ASI-R $=$ Anxiety Sensitivity Index-Revised, CT = cortical thickness, $\mathrm{SP}=$ specific phobia, ASI = 16-item Anxiety Sensitivity Index, SAP = specific animal phobia, GLM = general linear model, AS = anxiety sensitivity.

${ }^{a}$ It should be noted that all the studies have the same study design (cross-sectional, quantitative, nonexperimental).

${ }^{\mathrm{b}}$ Although not statistically significant, the authors found a positive correlation tendency between HARS score and CT in the left middle temporal cortex.

${ }^{\mathrm{c}}$ A similar tendency (negative association) was noted for the right postcentral cortex, although not statistically significant. 
Psychophysiological and Neurophysiological/Neuroimaging Measures. In terms of psychophysiological and neurophysiological measures of anxiety (Table 5), the SCR was the most commonly collected index, associated with one or more classical fear conditioning procedures (fear acquisition, fear extinction, fear recall, and/or fear renewal; $n=4$; Hartley et al., 2011; Milad et al., 2005, 2007; Winkelmann et al., 2016). One study collected heart rate variability during rest (Carnevali et al., 2019), and another collected fMRI data during a fear generalization task (Cha et al., 2014).

Three of the studies observed healthy individuals (Milad et al., 2005, 2007; Winkelmann et al., 2016) and two studies compared a sample of medication-free GAD patients with a group of HC (Carnevali et al., 2019; Cha et al., 2014). Another study did not report the status of the sample (Hartley et al., 2011).

Regarding the statistical analysis performed, the most common estimations used were the GLM ( $n=4$; Carnevali et al., 2019; Hartley et al., 2011; Milad et al., 2005; Winkelmann et al., 2016) and correlations ( $n=3$; Cha et al., 2014; Milad et al., 2005, 2007). Across studies, the authors controlled the following variables: age $(n=3$; Carnevali et al., 2019; Cha et al., 2014; Winkelmann et al., 2016), sex ( $n=1$; Winkelmann et al., 2016), experimental group ( $n=1$; Cha et al., 2014), intracranial volume ( $n=1$; Cha et al., 2014), global mean CT ( $n=1$; Carnevali et al., 2019), and the individual level of acquired fear across fear acquisition phases ( $n=1$; Winkelmann et al., 2016). Three studies did not report the control of variables (Hartley et al., 2011; Milad et al., 2005, 2007).

Regarding neurophysiological/neuroimaging measures, one study (Cha et al., 2014) found a statistically significant negative association between the CT in the left vmPFC and the vmPFC decreased discriminative blood-oxygen-level-dependent (BOLD) response. Also, the CT of the vmPFC was found to be a significant predictor of variance of the vmPFC fear generalization gradient, in a multiblock linear regression analysis.

Considering heart rate variability, one study (Carnevali et al., 2019) found that the natural logarithm of vagally-mediated heart rate variability $(\ln [\mathrm{vmHRV}])^{3}$ was predicted by the $\mathrm{CT}$ of the left caudal ACC, explaining $14 \%$ of the variance. This measure was also predicted by a significant interaction between Group and the CT of the left caudal ACC, with this interaction explaining $18 \%$ of the variance in the model. When post hoc tests were conducted, it showed that the CT of the left caudal ACC significantly predicted $\ln (\mathrm{vmHRV})$ in HC, but not in GAD patients.

Regarding the acquisition phase of the fear procedure, two studies found statistically significant effects for the association between CT and SCRs (Hartley et al., 2011; Milad et al.,

\footnotetext{
${ }^{3}$ In this study, the root mean square successive difference (RMSSD) was derived as a reliable index of vagally-mediated heart rate variability (vmHRV). A natural algorithm $(\ln [\mathrm{vmHRV}])$ was calculated to account for the non-normal distribution of vmHRV values.
} 
2007). Hartley et al. (2011) found that larger conditioned SCRs were associated with increased CT in the posterior insula/temporal operculum region (i.e., the CT of these regions was positively associated with fear acquisition). Although uncorrected, the $\mathrm{CT}$ of the following regions were also found to be positively associated with SCRs (Hartley et al., 2011): right and left medial temporal gyrus, right inferior temporal gyrus, right transverse temporal gyrus, left fusiform gyrus, right posterior cingulate sulcus, right medial occipital gyrus, and left inferior frontal gyrus. Milad et al. (2007), on the other hand, found that dACC thickness was positively associated with conditioned responses to the conditioned stimulus signaling fear $\left(\mathrm{CS}^{+}\right)$, as indexed by SCR.

In terms of the extinction learning phase, one study found a statistically significant negative association between the CT of three clusters (in the subgenual area of the right vmPFC, in the pars orbitalis of the left OFC, and in the medial frontal gyrus of the right OFC) and the SCRs collected during extinction learning. That is, individuals with a thicker vmPFC and OFC demonstrated faster extinction learning (Winkelmann et al., 2016).

Considering the extinction recall procedure, two studies found statistically significant effects (Hartley et al., 2011; Milad et al., 2005). Milad et al. (2005) found a negative association between the thickness of the right $\mathrm{mOFC}$ and the SCRs when processing the conditioned stimulus during extinction recall (one day after extinction learning). Hartley et al. (2011) found that the thickness of a small region of the left vmPFC was positively associated with extinction retention (individuals with a thicker cortex in this region exhibited greater fear inhibition), but this effect did not survive multiple comparisons correction.

Finally, Milad et al. (2005) found a significant positive correlation between CT of the mOFC and the left superior parietal cortex and the SCRs during the fear renewal phase. 


\section{Table 5}

Association Between CT and Other Anxiety Measures $(n=6)$

\begin{tabular}{|c|c|c|c|c|c|c|c|c|}
\hline \multirow[b]{2}{*}{ Study $^{\mathrm{a}}$} & \multirow{2}{*}{$\begin{array}{c}\text { Classical fear } \\
\text { conditioning } \\
\text { procedure }\end{array}$} & \multirow{2}{*}{$\begin{array}{c}\text { Other } \\
\text { measures of } \\
\text { anxiety }\end{array}$} & \multicolumn{5}{|c|}{ Sample information } & \multirow[b]{2}{*}{ Main findings } \\
\hline & & & Group $(n)$ & Comorbidity & $\begin{array}{c}\text { Medication } \\
\text { status }\end{array}$ & $\begin{array}{l}\text { Mean age } \\
\text { (SD) }\end{array}$ & $\begin{array}{c}\text { Sex } \\
(\mathrm{M} / \mathrm{F})\end{array}$ & \\
\hline
\end{tabular}






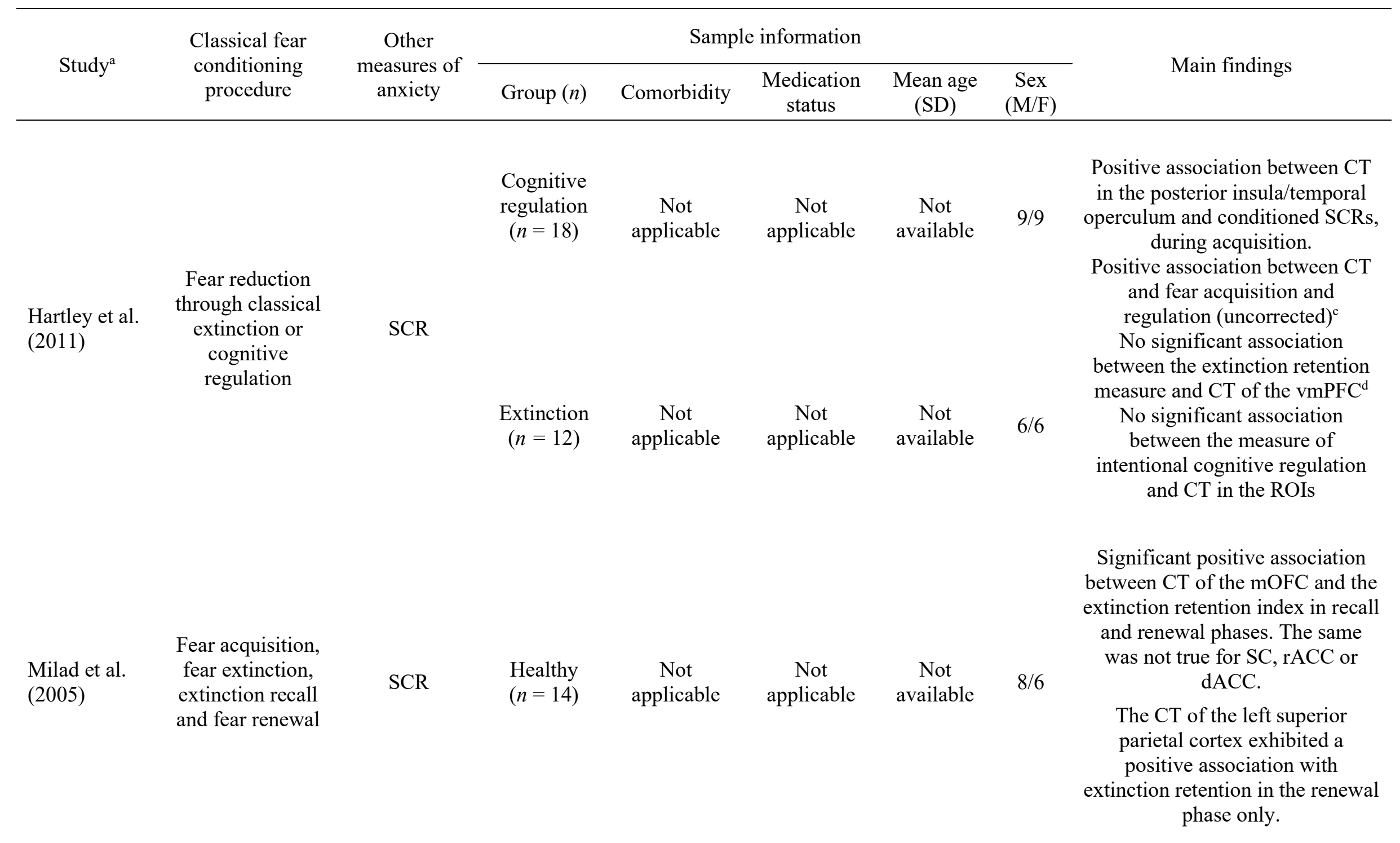




\begin{tabular}{|c|c|c|c|c|c|c|c|c|}
\hline \multirow{2}{*}{ Study ${ }^{\mathrm{a}}$} & \multirow{2}{*}{$\begin{array}{l}\text { Classical fear } \\
\text { conditioning } \\
\text { procedure }\end{array}$} & \multirow{2}{*}{$\begin{array}{l}\text { Other } \\
\text { measures of } \\
\text { anxiety }\end{array}$} & \multicolumn{5}{|c|}{ Sample information } & \multirow{2}{*}{ Main findings } \\
\hline & & & Group $(n)$ & Comorbidity & $\begin{array}{l}\text { Medication } \\
\text { status }\end{array}$ & $\begin{array}{l}\text { Mean age } \\
\text { (SD) }\end{array}$ & $\begin{array}{l}\text { Sex } \\
(\mathrm{M} / \mathrm{F})\end{array}$ & \\
\hline $\begin{array}{l}\text { Milad et al. } \\
\text { (2007) }\end{array}$ & $\begin{array}{l}\text { Fear acquisition } \\
\text { and extinction }\end{array}$ & SCR & $\begin{array}{l}\text { Healthy } \\
(n=14)\end{array}$ & $\begin{array}{c}\text { Not } \\
\text { applicable }\end{array}$ & $\begin{array}{c}\text { Not } \\
\text { applicable }\end{array}$ & $\begin{array}{c}\text { Not } \\
\text { available }\end{array}$ & $8 / 6$ & $\begin{array}{l}\text { Positive association between } \\
\text { CT in the dACC region and the } \\
\text { SCRs to the CS } \text { S }^{+} \text {but not to the } \\
\text { CS }^{-} \text {or differential SCR. }\end{array}$ \\
\hline $\begin{array}{l}\text { Winkelmann } \\
\text { et al. (2016) }\end{array}$ & $\begin{array}{l}\text { Fear acquisition } \\
\text { and extinction }\end{array}$ & SCR & $\begin{array}{l}\text { Replication } \\
\text { sample } \\
(n=53)\end{array}$ & $\begin{array}{c}\text { Not } \\
\text { applicable } \\
\text { (healthy) }\end{array}$ & $\begin{array}{c}\text { Not } \\
\text { applicable }\end{array}$ & $\begin{array}{l}21.83 \\
(2.98)\end{array}$ & $35 / 18$ & $\begin{array}{l}\text { Significant positive associations } \\
\text { between the differential SCRs of } \\
\text { the first extinction learning block } \\
\text { and the CT of three cortical } \\
\text { clusters: subgenual area of the } \\
\text { right vmPFC, the pars orbitalis of } \\
\text { the left orbitofrontal cortex; and } \\
\text { the medial frontal gyrus of the } \\
\text { right orbitofrontal cortex }\end{array}$ \\
\hline
\end{tabular}

Note. $\mathrm{M}=$ male, $\mathrm{F}=$ female, $\mathrm{GAD}=$ generalized anxiety disorder, $\mathrm{HC}=$ healthy control, $\ln (\mathrm{vmHRV})=$ natural logarithm of vagally-mediated heart rate variability, $\mathrm{CT}=$ cortical thickness, $\mathrm{ACC}=$ anterior cingulate cortex, $\mathrm{vmPFC}=$ ventromedial prefrontal cortex, $\mathrm{BOLD}=$ blood-oxygen-level-dependent, $\mathrm{fMRI}$ $=$ functional magnetic resonance imaging, $\mathrm{MDD}=$ major depressive disorder, $\mathrm{SCR}=$ skin conductance response, $\mathrm{ROI}=$ region-of-interest, $\mathrm{mOFC}=$ medial

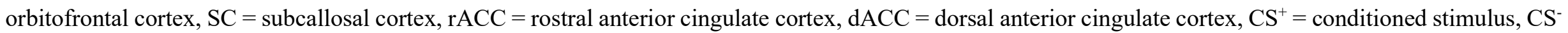
$=$ a stimulus that is not paired with the shock.

a. All the studies have the same study design (cross-sectional, nonexperimental and quantitative). 
b The non-normal distribution of vmHRV values was accounted for by calculating its natural logarithm, $\ln (\mathrm{vmHRV})$.

${ }^{\mathrm{c}}$ In the following regions: right posterior insula, right and left medial temporal gyrus, right inferior temporal gyrus, in the acquisition covariate of the cognitive regulation data set; left superior parietal lobule, left fusiform gyrus, left postcentral gyrus, left superior temporal gyrus, right precuneus, right and left medial temporal gyrus, in the regulation covariate of the cognitive regulation data set; right transverse temporal gyrus, left fusiform gyrus, right posterior cingulate sulcus, right medial occipital gyrus, left inferior frontal gyrus, right posterior insula/temporal operculum, in the acquisition covariate of the extinction data set; left superior temporal gyrus and left and right medial temporal gyrus, in the regulation covariate of the extinction data set.

${ }^{\mathrm{d}}$ A small region of the left vmPFC correlated positively with the index of extinction retention, using a lowered peak voxel threshold of $p=.003$. However, this correlation did not survive multiple comparisons correction. 


\section{Discussion}

\section{Summary of Evidence}

To the best of our knowledge, this scoping review is the first do address the association between $\mathrm{CT}$ and anxiety-related measures and to systematically identify and describe the studies about CT in what concerns MRI pre-processing and data analysis. The findings from the 17 identified studies suggest that there is a significant relationship between CT and several measures of anxiety across individual subjects, healthy or anxious.

In what concerns methodological options, the results show that the software FreeSurfer and its surface-based approach is the most used application for the measurement and analysis of CT. This may be due to the fact that FreeSurfer is an access-free, robust, reliable and accurate tool, added to its relatively easiness to navigate and its multiple features gathered in one unique software (Gransjøen, 2015).

However, the lack of a standard methodological practice and a standard practice for reporting the methods and results of CT analysis should be noted. The included studies differed in the methods undertaken, namely the statistical approach (whole-brain or ROI), the quality checking of the segmentation data, the Gaussian Kernel used, the scanner strength, and the sample size. Although most studies report controlling for variables identified in the literature as susceptible to influence the results (e.g., age and sex; Luders et al., 2006; Salat et al., 2004), the selection of controlled variables was diverse. This was also observed in the critical appraisal performed, where the main areas of inadequate quality pertain to the definition of exclusion criteria and cofounding factors. Finally, the included studies differed in the reporting of statistical results (corrected and uncorrected). As a result, caution is warranted when interpreting the findings, as there may be differences in the statistical power of the analysis and the resulting findings, increasing variability to the field.

Studies focusing on healthy samples might inform about potential vulnerability factors, even if cross-sectional. The reviewed findings indicate the possible existence of a significant relationship between higher anxiety scores, as assessed by self-report measures (STAI-trait scale, BAI and/or HARS), and differences in CT. Across studies, anxiety seems to be associated with a thickening of the left temporo-parietal regions (Blackmon et al., 2011) and thinning of the right and left mOFC (Carnevali et al., 2019; Kühn et al., 2011). Higher scores on the PSWQ scale, assessing pathological worry, were also associated with thinning of the left mOFC (Carnevali et al., 2019).

The temporo-parietal junction (TPJ) is thought to be involved in several processes, such as multisensory integration, social cognition and stimulus-driven attention functions (Eddy, 2016). In relation to adults, one study found that adolescents showed reduced activation in the TPJ during extinction recall compared to late extinction (Ganella et al., 2018). However, this region has not 
been notably studied considering anxiety disorders, to the best of our knowledge. Alternatively, inferior parietal regions have been found to be involved in contextual fear conditioning (Alvarez et al., 2008). Additionally, CT alterations have been reported in the left inferior parietal regions in anxiety disorders, namely a decreased thickness in GAD patients (Abdallah et al., 2012) and an increased thickness in SP patients (Rauch et al., 2004). With respect to the left posterior superior temporal cortex, this region has been implicated in social information processing, and seems to be dysfunctional in SAD patients (Gentili et al., 2008). Also, a significant positive association between trait anxiety and activation in this region has been previously reported (Fonzo et al., 2015). Taken together, these studies support the interest of the temporo-parietal regions in the development of anxiety disorders.

The mOFC, component of the vmPFC, has been previously associated with anxiety disorders in MRI and fMRI studies, namely in terms of its reduced volume and abnormal activation (Shin \& Liberzon, 2010; Xue et al., 2018). For example, its reduced function has been consistently associated with anxiety disorders, through various cognitive and symptom provocation paradigms (Craske et al., 2009; Milad \& Rauch, 2007). Considering the right mOFC, decreased CT of this region has been found in SAD (Syal et al., 2012) and GAD patients with comorbid MDD (Canu et al., 2015). In terms of CT alterations in the left mOFC, the results are discrepant. Whereas one study found increased CT in the left mOFC in GAD patients (Abdallah et al., 2012), another study found the opposite (Carnevali et al., 2019). This may be due to methodological differences of the studies or to other idiosyncratic variables at play.

The mOFC region is known to connect and project to other brain regions, such as the ventral striatum, the amygdala, the hypothalamus, and the hippocampus. These projections are suggested to be significantly involved in reward-oriented behavior and in emotional decision-making process. Additionally, the mOFC and the vmPFC have been implicated in fear extinction processes, such as extinction recall (Milad \& Rauch, 2007). Indeed, vmPFC thickness independently explained the individual variability in the vmPFC fear generalization gradient in one of the reviewed studies (Cha et al., 2014), and the increased thickness of the right mOFC was correlated with greater extinction memory, assessed by SCRs, in another study (Milad et al., 2005). Additionally, the finding that pathological worry (PSWQ) was associated with thinning of the left mOFC (Carnevali et al., 2019) supports the influence of autonomic responsivity, through suppression by the mOFC (Craske et al., 2009).

In what concerns the measurement instruments, higher scores in the STAI-trait scale were associated with decreased thickness in the right isthmus cingulate gyrus in HC (Carnevali et al., 2019). This region was also found to be thinner in GAD patients (Carnevali et al., 2019). In relation to BAI, an association between higher scores and increased thickness of left OFC regions was found (Blackmon et al., 2011). However, the left 1OFC region has been reported to be thinner in GAD 
(Abdallah et al., 2012; Maggioni et al., 2019), SAD (Zhao et al., 2017) and PD (Maggioni et al., 2019). Finally, higher scores in HARS were associated with increased thickness of the caudal and rostral ACC (Donzuso et al., 2014). These regions have been found to be thinner in GAD (Carnevali et al., 2019) and SAD (Zhao et al., 2017). Interestingly, vmHRV was also found to be significantly predicted by the CT of the left caudal ACC in HC (Carnevali et al., 2019). These findings point to an apparent contradiction regarding the association between $\mathrm{CT}$ and anxiety measures, in the sense that the direction of the effect seems to inverse in subjects with a clinical diagnosis or with the severity of anxiety.

Considering clinical samples, the selected studies did not allow for the assessment of vulnerability as they were cross-sectional. Thus, any assumptions concerning vulnerability factors or causality would be merely speculative. As such, the interpretation of the results will pertain to the association between the severity of anxiety and CT differences across the clinical conditions, keeping in mind that the associations may always be at least bidirectional within a complex system of factors. Current consensus is that the etiology of anxiety involves a multifactorial interaction between genetic vulnerability and environmental factors (e.g., stressful situations). Recently, epigenetic factors have also been implicated in the development of anxiety disorders. Exposure to stressful environmental conditions can trigger and encode persistent epigenetic reprogramming, which have the ability to change brain interactions with the environment (Règue-Guyon et al., 2018). Thus, other vulnerability factors may play a role in the development of anxiety disorders and act as mediators of the relationship between CT and anxiety, such as biological vulnerability factors (e.g., genetic, epigenetic, anxiety sensitivity). In order to better assess the role of CT in the development of anxiety disorders, longitudinal study designs or studies including monozygotic or dizygotic twins are needed.

Regarding the association between CT and clinical severity, an increased thickness of the right anterior insula was found to be associated with clinical severity in SAD and SP, through SPS and LSAS (Brühl et al., 2014) and ASI (Rosso et al., 2010), respectively. This is in accordance with the literature regarding the role of the insula in the mediation of fear and anxiety (Gogolla, 2017). In this sense, the evidence suggests a potential role of the insula as a translational component of anxiety disorders, considering a dimensional approach to psychopathology.

In relation to $\mathrm{PD}$, significant positive correlations were found in the left pars triangularis for both BAI, ASI-R and PDSS (Kang et al., 2017), as well as a negative correlation in the right temporal pole for ASI-R (Kang et al., 2017) and left rostral middle frontal cortex for PDSS (Asami et al, 2018). Functional and structural neuroimaging studies have also extensively revealed that the prefrontal and temporal cortex could be associated with the pathogenesis of PD (Coplan, \& Lydiard, 1998; Fontaine et al., 1990). For SAD, clinical severity (LSAS and SPS) was negatively associated with the thickness of the left postcentral cortex (Syal et al., 2012), and positively associated with 
the thickness of the parahippocampal gyrus (Brühl et al., 2014). This is in line with studies in SAD that have found that mental imagery resulted in activity in the left postcentral gyrus (Kilts et al., 2006), as well as greater BOLD responses observed in the parahippocampal gyrus (Goldin et al., 2009). Considering GAD, clinical severity (STAI and PSWQ) appears to be associated with decreased thickness of the left mOFC and right isthmus cingulate gyrus (Carnevali et al., 2019). Conversely, mOFC volumes were previously positively associated with worry scores in GAD patients (Mohlman et al., 2009). Future studies should attempt to assess and compare these different morphological outcomes (e.g., volume, thickness, surface area).

It should be noted that divergent results occurred across the different clinical conditions. That is, the relationship between STAI scores and CT was significant in GAD patients in the left mOFC and right isthmus cingulate gyrus (Carnevali et al., 2019), but not for SAD (Brühl et al., 2014). Future replication studies are warranted to evaluate these discrepancies. Taking these associations into account, altered CT in the identified regions may be a neural correlate of the disorder in question but not a psychopathological dimension.

Overall, two factors should be accounted when interpreting the available findings across studies. First, the majority of included patients in clinical samples were taking medication. Although the effects of a particular medication on CT have not been fully elucidated, changes in CT due to long-term medication in-take cannot be ruled out as potential cofounders. Second, there was a high rate of comorbidity in clinical samples, namely with MDD. Patients who present anxiety and depression comorbidity are known to show increased clinical severity and higher rates of chronicity (Hirschfeld, 2001). Although there is a significant overlap in the neural system disfunction of MDD and anxiety disorders (Hamilton et al., 2015), they have unique features as well. This comorbidity could potentially cofound the present findings. However, considering the present direction of clinical neuroscience, the specific variations between studies might demonstrate the contribution of multiple structural abnormalities to the development of the broad categories of affective disorders (Williams, 2016).

In this way, another gap in knowledge pertains to how comorbidities may impact the association between CT and anxiety, either in terms of mood disorders (e.g., MDD) or other anxiety disorders. Due to the strong evidence of similar etiological and maintenance processes underlying depressive and anxious psychopathology, transdiagnostic processes should be considered. These disorders share similar genetic and environmental risk factors (e.g., "triple vulnerabilities" theory; Barlow et al., 2016). Therefore, changes in CT in specific regions of fundamental functional systems could potentially be considered as a translational component of vulnerability. This is compatible with the Research Domain Criteria (RDoC) framework, focused on the identification of common and core mechanisms underlying multiple disorders, such as cognition, negative affect, and arousal (Cuthbert, 2014; Newby et al., 2015). 
Anxiety disorders can also be conceptualized as involving disturbances in the fear circuitry responsible for the fear response (Graham \& Milad, 2011). Taken together, the reviewed findings point to differences in $\mathrm{CT}$ in several brain regions that are associated with distinctive phases of the fear response, and some contributing simultaneously to more than one. Firstly, an increased thickness of the posterior insula/temporal operculum and of the dACC appears to be associated with fear learning (Hartley et al., 2011; Milad et al., 2007). Secondly, the thickness of the vmPFC was associated with both faster extinction learning (Winkelmann et al., 2016) and larger extinction retention (Hartley et al., 2011). Thirdly, the mOFC seems to be negatively involved in extinction recall and positively involved in fear renewal phases (Milad et al., 2005). Finally, the thickness of the OFC is positively associated with fear extinction learning (Winkelmann et al., 2016). These regions have been previously reported in the literature as being activated during fear conditioning processes (Sehlmeyer et al., 2009) and represent areas of particular interest for the development and treatment of anxiety disorders, as differences in CT may interfere with treatment response.

Overall, CT appears to be a precursor of the development of anxiety disorders, as well as a contributing factor to their clinical severity. As previously mentioned, it is not possible to dismiss the possibility of a bidirectional relationship between these two variables. Replication studies are warranted to assess the divergent results noted, as well as studies evaluating the association between CT differences and other anxiety measures, such as the Anxiety Attitude and Belief Scale (Brown et al., 2000). Finally, studies should also assess if differences in CT may be related to specific components of anxiety (e.g., respiratory somatic symptoms, worry, apprehension) and the wellknown desynchrony presented between measures (McLean \& Woody, 2001). This is in line with evidence that shows conflicting results concerning the association between CT and cognitive, neural, autonomic arousal, and behavior measures of anxiety.

\section{Limitations}

This study is not without limitations. First, the search was limited to English and Portuguese languages. Second, as it is a review, the analysis was limited to the information reported in the included studies. However, a quality assessment of the reviewed articles was performed and found that the majority of the included articles were of a high quality. Third, due to being out of the scope of the review, not all anxiety measures were included (e.g., neuroendocrine, genetic, and epigenetic). Also, we did not search grey literature. Finally, the present scoping review could be extended to other processes underlying anxiety, as we focused solely on fear conditioning processes.

\section{Conclusions and Recommendations}

Alterations in CT may act as a vulnerability factor for the development of anxiety disorders, as seen by findings in healthy population studies and in studies concerning fear conditioning 
processes. Several brain regions seem to be of interest in clinical anxiety, namely the mOFC, the vmPFC, the insula, the TPJ and the ACC. These findings may enhance our understanding of the role of the $\mathrm{CT}$ in developing anxiety disorders, although more studies are warranted to confirm the findings of some of the reviewed studies.

\section{Implications for Future Research}

This scoping review aimed to summarize the main methods and findings pertaining to the association between $\mathrm{CT}$ and measures of anxiety. The findings here reported may support the understanding of the mechanisms underlying the development of anxiety disorders, namely the association between CT and measures of anxiety, and inform the design of future studies.

\section{Conflicts and Acknowledgements}

\section{Conflicts of Interest}

None exist.

\section{Funding}

This research did not receive particular funding.

\section{Acknowledgements}

We thank Sara Morgado for her contribution in the selection and revision of articles, extraction of data, and overall input regarding the process of the scoping review. We thank Raquel Guiomar for her assistance during the various stages of the review preparation and insightful discussions. We thank Joana Coutinho, Marie-Anne Vanderhasselt, Debby Klooster, and Frauke Nees for their expert knowledge that supported decisions concerning the focus of the scoping review. Finally, this review was conducted as part of a project supported by the Foundation for Science and Technology, Portugal and Program COMPETE [grant number PTDC/MHC$\mathrm{PAP} / 5618 / 2014$ compete2020.]]http://www.poci-compete2020.]. 


\section{References}

Abdallah, C. G., Coplan, J. D., Jackowski, A., Sato, J. R., Mao, X., Shungu, D. C., \& Mathew, S. J. (2012). Riluzole effect on occipital cortex: A structural and spectroscopy pilot study. Neuroscience Letters, 530(1), 103-107. https://doi.org/10.1016/j.neulet.2012.09.054

Alvarez, R. P., Biggs, A., Chen, G., Pine, D. S., \& Grillon, C. (2008). Contextual fear conditioning in humans: Cortical-hippocampal and amygdala contributions. The Journal of Neuroscience, 28(24), 6211-6219. https://doi.org/10.1523/JNEUROSCI.1246-08.2008

American Psychiatric Association. (2013). Diagnostic and statistical manual of mental disorders (5th ed.). https://doi.org/10.1176/appi.books.9780890425596

Arksey, H., \& O'Malley, L. (2005). Scoping studies: Towards a methodological framework. International Journal of Social Research Methodology, 8(1), 19-32. https://doi.org/10.1080/1364557032000119616

Asami, T., Takaishi, M., Nakamura, R., Yoshida, H., Yoshimi, A., Whitford, T. J., Inoue, T., \& Hirayasu, Y. (2018). Cortical thickness reductions in the middle frontal cortex in patients with panic disorder. Journal of Affective Disorders, 240, 199-202. https://doi.org/10.1016/j.jad.2018.07.064

Barlow, D. H., Allen, L. B., \& Choate, M. L. (2016). Toward a unified treatment for emotional disorders - Republished article. Behavior Therapy, 47(6), 838-853. https://doi.org/10.1016/j.beth.2016.11.005

Baxter, A. J., Scott, K. M., Vos, T., \& Whiteford, H. A. (2013). Global prevalence of anxiety disorders: A systematic review and meta-regression. Psychological Medicine, 43(5), 897910. https://doi.org/10.1017/S003329171200147X

Beck, A. T., Epstein, N., Brown, G., \& Steer, R. A. (1988). An inventory for measuring clinical anxiety: Psychometric properties. Journal of Consulting and Clinical Psychology, 56(6), 893-897. https://doi.org/10.1037/0022-006X.56.6.893

Blackmon, K., Barr, W. B., Carlson, C., Devinsky, O., DuBois, J., Pogash, D., Quinn, B. T., Kuzniecky, R., Halgren, E., \& Thesen, T. (2011). Structural evidence for involvement of a left amygdala-orbitofrontal network in subclinical anxiety. Psychiatry Research: Neuroimaging, 194(3), 296-303. https://doi.org/10.1016/j.pscychresns.2011.05.007 
Brown, G. P., Craske, M. G., Tata, P., Rassovsky, Y., \& Tsao, J. C. I. (2000). The Anxiety Attitude and Belief Scale: Initial psychometric properties in an undergraduate sample. Clinical Psychology \& Psychotherapy, 7(3), 230-239. https://doi.org/10.1002/10990879(200007)7:3<230::AID-CPP239>3.0.CO;2-Y

Brühl, A. B., Hänggi, J., Baur, V., Rufer, M., Delsignore, A., Weidt, S., Jäncke, L., \& Herwig, U. (2014). Increased cortical thickness in a frontoparietal network in social anxiety disorder. Human Brain Mapping, 35(7), 2966-2977. https://doi.org/10.1002/hbm.22378

Canu, E., Kostić, M., Agosta, F., Munjiza, A., Ferraro, P. M., Pesic, D., Copetti, M., Peljto, A., Tosevski, D. L., \& Filippi, M. (2015). Brain structural abnormalities in patients with major depression with or without generalized anxiety disorder comorbidity. Journal of Neurology, 262(5), 1255-1265. https://doi.org/10.1007/s00415-015-7701-z

Carnevali, L., Mancini, M., Koenig, J., Makovac, E., Watson, D. R., Meeten, F., Critchley, H. D., \& Ottaviani, C. (2019). Cortical morphometric predictors of autonomic dysfunction in generalized anxiety disorder. Autonomic Neuroscience, 217, 41-48. https://doi.org/10.1016/j.autneu.2019.01.001

Cha, J., Greenberg, T., Carlson, J. M., Dedora, D. J., Hajcak, G., \& Mujica-Parodi, L. R. (2014). Circuit-wide structural and functional measures predict ventromedial prefrontal cortex fear generalization: Implications for generalized anxiety disorder. The Journal of Neuroscience, 34(11), 4043-4053. https://doi.org/10.1523/JNEUROSCI.3372-13.2014

Coplan, J. D., \& Lydiard, R. B. (1998). Brain circuits in panic disorder. Biological Psychiatry, 44(12), 1264-1276. https://doi.org/10.1016/s0006-3223(98)00300-x

Craske, M. G., Rauch, S. L., Ursano, R., Prenoveau, J., Pine, D. S., \& Zinbarg, R. E. (2009). What is an anxiety disorder?. Depression and Anxiety, 26(12), 1066-1085. https://doi.org/10.1002/da.20633

Cuthbert, B. N. (2014). The RDoC framework: Facilitating transition from ICD/DSM to dimensional approaches that integrate neuroscience and psychopathology. World Psychiatry, 13(1), 28-35. https://doi.org/10.1002/wps.20087

Das, S. R., Avants, B. B., Grossman, M., \& Gee, J. C. (2009). Registration based cortical thickness measurement. NeuroImage, $\quad$ 45(3), 867-879. https://doi.org/10.1016/j.neuroimage.2008.12.016 
Donzuso, G., Cerasa, A., Gioia, M. C., Caracciolo, M., \& Quattrone, A. (2014). The neuroanatomical correlates of anxiety in a healthy population: Differences between the State-Trait Anxiety Inventory and the Hamilton Anxiety Rating Scale. Brain and Behavior, 4(4), 504-514. https://doi.org/10.1002/brb3.232

Duval, E. R., Javanbakht, A., \& Liberzon, I. (2015). Neural circuits in anxiety and stress disorders: A focused review. Therapeutics and Clinical Risk Management, 11, 115-126. https://doi.org/10.2147/TCRM.S48528

Eddy, C. M. (2016). The junction between self and other? Temporo-parietal dysfunction in $\begin{array}{llll}\text { neuropsychiatry. } & \text { Neuropsychologia, } & 89, & 465-477\end{array}$ https://doi.org/10.1016/j.neuropsychologia.2016.07.030

Field, A. (2009). Discovering statistics using SPSS (and sex and drugs and rock 'n' roll) (3rd ed.). Sage. http://sro.sussex.ac.uk/id/eprint/14911

Fischl, B., \& Dale, A. M. (2000). Measuring the thickness of the human cerebral cortex from magnetic resonance images. PNAS, 97(20), 11050-11055. https://doi.org/10.1073/pnas.200033797

Fontaine, R., Breton, G., Déry, R., Fontaine, S., \& Elie, R. (1990). Temporal lobe abnormalities in panic disorder: An MRI study. Biological Psychiatry, 27(3), 304-310. https://doi.org/10.1016/0006-3223(90)90004-1

Fonzo, G. A., Ramsawh, H. J., Flagan, T. M., Sullivan, S. G., Letamendi, A., Simmons, A. N., Paulus, M. P., \& Stein, M. B. (2015). Common and disorder-specific neural responses to emotional faces in generalised anxiety, social anxiety and panic disorders. The British Journal of Psychiatry, 206(3), 206-215. https://doi.org/10.1192/bjp.bp.114.149880

Ganella, D. E., Drummond, K. D., Ganella, E. P., Whittle, S., \& Kim, J. H. (2018). Extinction of conditioned fear in adolescents and adults: A human fMRI study. Frontiers in Human Neuroscience, 11, Article 647. https://doi.org/10.3389/fnhum.2017.00647

Gentili, C., Gobbini, M. I., Ricciardi, E., Vanello, N., Pietrini, P., Haxby, J. V., \& Guazzelli, M. (2008). Differential modulation of neural activity throughout the distributed neural system for face perception in patients with social phobia and healthy subjects. Brain Research Bulletin, 77(5), 286-292. https://doi.org/10.1016/j.brainresbull.2008.08.003 
Gogolla, N. (2017). The insular cortex. Current Biology, 27(12), R580-R586. https://doi.org/10.1016/j.cub.2017.05.010

Gold, A. L., Steuber, E. R., White, L. K., Pacheco, J., Sachs, J. F., Pagliaccio, D., Berman, E., Leibenluft, E., \& Pine, D. S. (2017). Cortical thickness and subcortical gray matter volume in pediatric anxiety disorders. Neuropsychopharmacology, 42(12), 2423-2433. https://doi.org/10.1038/npp.2017.83

Goldin, P. R., Manber, T., Hakimi, S., Canli, T., \& Gross, J. J. (2009). Neural bases of social anxiety disorder: Emotional reactivity and cognitive regulation during social and physical threat. Archives of General Psychiatry, 66(2), 170-180. https://doi.org/10.1001/archgenpsychiatry.2008.525

Graham, B. M., \& Milad, M. R. (2011). The study of fear extinction: Implications for anxiety disorders. American Journal of Psychiatry, 168(12), 1255-1265. https://doi.org/10.1176/appi.ajp.2011.11040557

Gransjøen, A. M. (2015). Cortical thickness analysis - The methods. Radiography Open, 2(1), $52-$ 64. https://doi.org/10.7577/radopen.1529

Hamilton, J. P., Chen, M. C., Waugh, C. E., Joormann, J., \& Gotlib, I. H. (2015). Distinctive and common neural underpinnings of major depression, social anxiety, and their comorbidity. Social Cognitive and Affective Neuroscience, 10(4), 552-560. https://doi.org/10.1093/scan/nsu084

Hanford, L. C., Nazarov, A., Hall, G. B., \& Sassi, R. B. (2016). Cortical thickness in bipolar disorder: A systematic review. Bipolar Disorders, 18(1), 4-18. https://doi.org/10.1111/bdi.12362

Hartley, C. A., Fischl, B., \& Phelps, E. A. (2011). Brain structure correlates of individual differences in the acquisition and inhibition of conditioned fear. Cerebral Cortex, 21(9), 1954-1962. https://doi.org/10.1093/cercor/bhq253

Hirschfeld, R. M. A. (2001). The comorbidity of major depression and anxiety disorders: Recognition and management in primary care. Primary Care Companion to The Journal of Clinical Psychiatry, 3(6), 244-254. https://doi.org/10.4088/pcc.v03n0609 
Hutton, C., Vita, E. D., Ashburner, J., Deichmann, R., \& Turner, R. (2008). Voxel-based cortical thickness measurements in MRI. NeuroImage, 40(4), 1701-1710. https://doi.org/10.1016/j.neuroimage.2008.01.027

Hutton, C., Draganski, B., Ashburner, J., \& Weiskopf, N. (2009). A comparison between voxelbased cortical thickness and voxel-based morphometry in normal aging. NeuroImage, 48(2), 371-380. https://doi.org/10.1016/j.neuroimage.2009.06.043

Kang, E.-K., Lee, K. S., \& Lee, S.-H. (2017). Reduced cortical thickness in the temporal pole, insula, and pars triangularis in patients with panic disorder. Yonsei Medical Journal, 58(5), 1018-1024. https://doi.org/10.3349/ymj.2017.58.5.1018

Kilts, C. D., Kelsey, J. E., Knight, B., Ely, T. D., Bowman, F. D., Gross, R. E., Selvig, A., Gordon, A., Newport, D. J., \& Nemeroff, C. B. (2006). The neural correlates of social anxiety disorder and response to pharmacotherapy. Neuropsychopharmacology, 31(10), 22432253. https://doi.org/10.1038/sj.npp.1301053

Kühn, S., Schubert, F., \& Gallinat, J. (2011). Structural correlates of trait anxiety: Reduced thickness in medial orbitofrontal cortex accompanied by volume increase in nucleus accumbens. Journal of Affective Disorders, 134(1-3), 315-319. https://doi.org/10.1016/j.jad.2011.06.003

Landis, J. R., \& Koch, G. G. (1977). The measurement of observer agreement for categorical data. Biometrics, 33(1), 159-174. https://doi.org/10.2307/2529310

Levac, D., Colquhoun, H., \& O’Brien, K. K. (2010). Scoping studies: Advancing the methodology. Implementation Science, 5, Article 69. https://doi.org/10.1186/1748-5908-5-69

Lonsdorf, T. B., Menz, M. M., Andreatta, M., Fullana, M. A., Golkar, A., Haaker, J., Heitland, I., Hermann, A., Kuhn, M., Kruse, O., Drexler, S. M., Meulders, A., Nees, F., Pittig, A., Richter, J., Römer, S., Shiban, Y., Schmitz, A., Straube, B., .. Merz, C. J. (2017). Don’t fear 'fear conditioning': Methodological considerations for the design and analysis of studies on human fear acquisition, extinction, and return of fear. Neuroscience \& Biobehavioral Reviews, 77, 247-285. https://doi.org/10.1016/j.neubiorev.2017.02.026

Luders, E., Narr, K. L., Thompson, P. M., Rex, D. E., Woods, R. P., DeLuca, H., Jancke, L., \& Toga, A. W. (2006). Gender effects on cortical thickness and the influence of scaling. Human Brain Mapping, 27(4), 314-324. https://doi.org/10.1002/hbm.20187 
Maggioni, E., Delvecchio, G., Grottaroli, M., Garzitto, M., Piccin, S., Bonivento, C., Maieron, M., D’Agostini, S., Perna, G., Balestrieri, M., \& Brambilla, P. (2019). Common and different neural markers in major depression and anxiety disorders: A pilot structural magnetic resonance imaging study. Psychiatry Research: Neuroimaging, 290, 42-50. https://doi.org/10.1016/j.pscychresns.2019.06.006

Makris, N., Kaiser, J., Haselgrove, C., Seidman, L. J., Biederman, J., Boriel, D., Valera, E. M., Papadimitriou, G. M., Fischl, B., Caviness, V. S., \& Kennedy, D. N. (2006). Human cerebral cortex: A system for the integration of volume- and surface-based representations. NeuroImage, 33(1), 139-153. https://doi.org/10.1016/j.neuroimage.2006.04.220

McLean, P. D., \& Woody, S. R. (2001). Specific fears and phobias. In Anxiety disorders in adults: An evidence-based approach to psychological treatment (pp. 48-83). Oxford University Press. https://doi.org/10.1093/med:psych/9780195116250.003.0003

Milad, M. R., \& Rauch, S. L. (2007). The role of the orbitofrontal cortex in anxiety disorders. Annals of the New York Academy of Sciences, 1121(1), 546-561. https://doi.org/10.1196/annals.1401.006

Milad, M. R., Quinn, B. T., Pitman, R. K., Orr, S. P., Fischl, B., \& Rauch, S. L. (2005). Thickness of ventromedial prefrontal cortex in humans is correlated with extinction memory. PNAS, 102(30), 10706-10711. https://doi.org/10.1073/pnas.0502441102

Milad, M. R., Quirk, G. J., Pitman, R. K., Orr, S. P., Fischl, B., \& Rauch, S. L. (2007). A role for the human dorsal anterior cingulate cortex in fear expression. Biological Psychiatry, 62(10), 1191-1194. https://doi.org/10.1016/j.biopsych.2007.04.032

Molent, C., Maggioni, E., Cecchetto, F., Garzitto, M., Piccin, S., Bonivento, C., Maieron, M., D’Agostini, S., Balestrieri, M., Perna, G., Altamura, A. C., \& Brambilla, P. (2018). Reduced cortical thickness and increased gyrification in generalized anxiety disorder: A 3 T MRI study. Psychological Medicine, 48(12), 2001-2010. https://doi.org/10.1017/S003329171700352X

Mohlman, J., Price, R. B., Eldreth, D. A., Chazin, D., Glover, D. M., \& Kates, W. R. (2009). The relation of worry to prefrontal cortex volume in older adults with and without generalized anxiety disorder. Psychiatry Research, 173(2), 121-127. https://doi.org/10.1016/j.pscychresns.2008.09.010 
Moola, S., Munn, Z., Tufanaru, C., Aromataris, E., Sears, K., Sfetcu, R., Currie, M., Lisy, K., Qureshi, R., Mattis, P., \& Mu, P. (2020). Chapter 7: Systematic reviews of etiology and risk. In E. Aromataris \& Z. Munn (Eds.), JBI Manual for Evidence Synthesis (pp. 219-272). The Joanna Briggs Institute. https://doi.org/10.46658/JBIMES-20-08

Newby, J. M., McKinnon, A., Kuyken, W., Gilbody, S., \& Dalgleish, T. (2015). Systematic review and meta-analysis of transdiagnostic psychological treatments for anxiety and depressive disorders in adulthood. Clinical Psychology Review, 40, 91-110. https://doi.org/10.1016/j.cpr.2015.06.002

Ouzzani, M., Hammady, H., Fedorowicz, Z., \& Elmagarmid, A. (2016). Rayyan - A web and mobile app for systematic reviews. Systematic Reviews, 5(1), Article 210. https://doi.org/10.1186/s13643-016-0384-4

Panizzon, M. S., Fennema-Notestine, C., Eyler, L. T., Jernigan, T. L., Prom-Wormley, E., Neale, M., Jacobson, K., Lyons, M. J., Grant, M. D., Franz, C. E., Xian, H., Tsuang, M., Fischl, B., Seidman, L., Dale, A., \& Kremen, W. S. (2009). Distinct genetic influences on cortical surface area and cortical thickness. Cerebral Cortex, 19(11), 2728-2735. https://doi.org/10.1093/cercor/bhp026

Peters, M. D. J., Godfrey, C. M., McInerney, P., Munn, Z., Tricco, A. C., \& Khalil, H. (2020). Chapter 11: Scoping Reviews. In E. Aromataris \& Z. Munn (Eds.), JBI Manual for Evidence Synthesis (pp. 407-452). https://doi.org/10.46658/JBIRM-20-01

Rakic, P. (1988). Specification of cerebral cortical areas. Science, 241(4862), 170-176. https://doi.org/10.1126/science.3291116

Rakic, P. (2007). The radial edifice of cortical architecture: From neuronal silhouettes to genetic engineering. Brain Research Reviews, 55(2), 204-219. https://doi.org/10.1016/j.brainresrev.2007.02.010

Rauch, S. L., Wright, C. I., Martis, B., Busa, E., McMullin, K. G., Shin, L. M., Dale, A. M., \& Fischl, B. (2004). A magnetic resonance imaging study of cortical thickness in animal $\begin{array}{llll}\text { phobia. } & \text { Biological }\end{array}$ https://doi.org/10.1016/j.biopsych.2003.12.022

Règue-Guyon, M., Lanfumey, L., \& Mongeau, R. (2018). Neuroepigenetics of neurotrophin signaling: Neurobiology of anxiety and affective disorders. In B. P. F. Rutten (Ed.), 
Progress in Molecular Biology and Translational Science (Vol. 158, pp. 159-193). Academic Press. https://doi.org/10.1016/bs.pmbts.2018.03.002

Rosso, I. M., Makris, N., Britton, J. C., Price, L. M., Gold, A. L., Zai, D., Bruyere, J., Deckersbach, T., Killgore, W. D. S., \& Rauch, S. L. (2010). Anxiety sensitivity correlates with two indices of right anterior insula structure in specific animal phobia. Depression and Anxiety, 27(12), 1104-1110. https://doi.org/10.1002/da.20765

Salat, D. H., Buckner, R. L., Snyder, A. Z., Greve, D. N., Desikan, R. S. R., Busa, E., Morris, J. C., Dale, A. M., \& Fischl, B. (2004). Thinning of the cerebral cortex in aging. Cerebral Cortex, 14(7), 721-730. https://doi.org/10.1093/cercor/bhh032

Sehlmeyer, C., Schöning, S., Zwitserlood, P., Pfleiderer, B., Kircher, T., Arolt, V., \& Konrad, C. (2009). Human fear conditioning and extinction in neuroimaging: A systematic review. PloS One, 4(6), Article e5865. https://doi.org/10.1371/journal.pone.0005865

Shin, L. M., \& Liberzon, I. (2010). The neurocircuitry of fear, stress, and anxiety disorders. Neuropsychopharmacology, 35(1), 169-191. https://doi.org/10.1038/npp.2009.83

Singh, V., Chertkow, H., Lerch, J. P., Evans, A. C., Dorr, A. E., \& Kabani, N. J. (2006). Spatial patterns of cortical thinning in mild cognitive impairment and Alzheimer's disease. Brain, 129(11), 2885-2893. https://doi.org/10.1093/brain/aw1256

Sobral, M. A. C., Ganho-Ávila, A., Guiomar, R. N. R. M., \& Moreira, H. T. C. (2020). Association between cortical thickness and anxiety measures: A scoping review protocol. https://doi.org/10.17605/OSF.IO/NZPDW

Spielberger, C. D., Gorsuch, R. L., Lushene, R., Vagg, P. R., \& Jacobs, G. A. (1983). Manual for the State-Trait Anxiety Inventory. Consulting Psychologists Press.

Suh, J. S., Schneider, M. A., Minuzzi, L., MacQueen, G. M., Strother, S. C., Kennedy, S. H., \& Frey, B. N. (2019). Cortical thickness in major depressive disorder: A systematic review and meta-analysis. Progress in Neuro-Psychopharmacology and Biological Psychiatry, 88 , 287-302. https://doi.org/10.1016/j.pnpbp.2018.08.008

Syal, S., Hattingh, C. J., Fouché, J.-P., Spottiswoode, B., Carey, P. D., Lochner, C., \& Stein, D. J. (2012). Grey matter abnormalities in social anxiety disorder: A pilot study. Metabolic Brain Disease, 27(3), 299-309. https://doi.org/10.1007/s11011-012-9299-5 
Tinoco-González, D., Fullana, M. A., Torrents-Rodas, D., Bonillo, A., Vervliet, B., Blasco, M. J., Farré, M., \& Torrubia, R. (2015). Conditioned fear acquisition and generalization in generalized anxiety disorder. Behavior Therapy, 46(5), 627-639. https://doi.org/10.1016/j.beth.2014.12.004

Tricco, A. C., Lillie, E., Zarin, W., O’Brien, K. K., Colquhoun, H., Levac, D., Moher, D., Peters, M. D. J., Horsley, T., Weeks, L., Hempel, S., Akl, E. A., Chang, C., McGowan, J., Stewart, L., Hartling, L., Aldcroft, A., Wilson, M. G., Garritty, C., ... Straus, S. E. (2018). PRISMA extension for scoping reviews (PRISMA-ScR): Checklist and explanation. Annals of Internal Medicine, 169(7), 467-473. https://doi.org/10.7326/M18-0850

Wagstyl, K., \& Lerch, J. P. (2018). Cortical thickness. In G. Spalletta, F. Piras, \& T. Gili (Eds.), Brain Morphometry (Vol. 136, pp. 35-49). Humana Press. https://doi.org/10.1007/978-14939-7647-8_3

Williams, L. M. (2016). Precision psychiatry: A neural circuit taxonomy for depression and anxiety. The Lancet Psychiatry, 3(5), 472-480. https://doi.org/10.1016/S2215-0366(15)00579-9

Winkelmann, T., Grimm, O., Pohlack, S. T., Nees, F., Cacciaglia, R., Dinu-Biringer, R., Steiger, F., Wicking, M., Ruttorf, M., Schad, L. R., \& Flor, H. (2016). Brain morphology correlates of interindividual differences in conditioned fear acquisition and extinction learning. Brain Structure and Function, 221(4), 1927-1937. https://doi.org/10.1007/s00429-015-1013-z

Winkler, A. M., Kochunov, P., Blangero, J., Almasy, L., Zilles, K., Fox, P. T., Duggirala, R., \& Glahn, D. C. (2010). Cortical thickness or grey matter volume? The importance of selecting the phenotype for imaging genetics studies. NeuroImage, 53(3), 1135-1146. https://doi.org/10.1016/j.neuroimage.2009.12.028

Xue, S., Lee, T., \& Guo, Y. (2018). Spontaneous activity in medial orbitofrontal cortex correlates with trait anxiety in healthy male adults. Journal of Zhejiang University SCIENCE BBiomedicine \& Biotechnology, 19(8), 643-653. https://doi.org/10.1631/jzus.B1700481

Zhao, Y., Chen, L., Zhang, W., Xiao, Y., Shah, C., Zhu, H., Yuan, M., Sun, H., Yue, Q., Jia, Z., Zhang, W., Kuang, W., Gong, Q., \& Lui, S. (2017). Gray matter abnormalities in noncomorbid medication-naive patients with major depressive disorder or social anxiety disorder. EBioMedicine, 21, 228-235. https://doi.org/10.1016/j.ebiom.2017.06.013 


\section{Appendix A}

\section{Scoping Review Protocol}

\section{Protocol for a Scoping Review}

- Title: Association Between Cortical Thickness and Measures of Anxiety: A Scoping Review

- Registration: Open Science Framework

- Start Date: September 2019

- Anticipated Completion Date: July 2020

- Funding: -

- Stage of review at time of the submission: Data analysis

- Ethics and Dissemination: As the scoping review entails the mapping of the existing evidence through the synthetization of information of available studies, no data was further collected, and no ethical approval was required. Results will be disseminated through scientific outcomes such as publication of the scoping review in peer reviewed international journals.

\section{Theoretical Framework}

Anxiety disorders are a significant condition in general population, affecting up to one third during their lifetime (Bandelow \& Michaelis, 2015). According to the Diagnostic and Statistical Manual of Mental Disorders (5th ed.; DSM-5; American Psychiatric Association [APA], 2013), these disorders include generalized anxiety disorder, panic disorder, specific phobias, agoraphobia, social anxiety disorder (social phobia), separation anxiety disorder, among others. In DSM-5, obsessive-compulsive disorder (OCD), acute stress disorder, and posttraumatic stress disorder (PTSD) are no longer considered anxiety disorders (APA, 2013).

Anxiety disorders are an excessive reaction of fear or anxiety, characterized by cognitive, behavioral, somatic, and emotional components (APA, 2013). These components can be assessed through different types of measures, namely clinical self-report (e.g., Beck Anxiety Inventory [BAI; Beck et al., 1988]), psychophysiological (e.g., galvanic skin response, heart rate variability), neuroendocrine (e.g., cortisol levels), behavioral (e.g., reaction time), functional neuroimaging (e.g., functional magnetic resonance imaging [fMRI], electroencephalography), genetic (e.g., 5HTTLPR), and epigenetic measures (e.g., serotonin pattern of methylation). However, the meaning of the assessment scores across some measures of anxiety remains unclear, such as The State-Trait Anxiety Inventory (STAI; Spielberger et al., 1983) and the Hamilton scale for anxiety (HARS; Hamilton, 1959). That is, what their scores reflect is still uncertain (do they measure similar aspects 
of anxiety or do the scores reflect different aspects of the biological mechanisms underlying anxiety?; Donzuso et al., 2014).

Abnormal fear conditioning processes, such as fear acquisition, are one of the mechanisms to have been found to be associated with the pathogenesis of anxiety disorders (Tinoco-González et al., 2015). Nonetheless, the interaction between biopsychosocial risk factors to the development of anxiety disorders is not yet clear. As a result, translational neuroscience research has aimed to identify brain structure alterations and fundamental circuits underlying anxiety disorders, in order to better predict treatment response and guide the development of new treatments (Gold et al., 2017; Shin \& Liberzon, 2010). Thus, the biomarkers of anxiety and its related processes have been increasingly researched.

Cortical thickness (CT) could potentially be considered as a biomarker of interest in the study of anxiety disorders. CT is commonly defined as the shortest distance between two corresponding points on the pial and the white matter boundaries of the neocortex (Das et al., 2009). The thickness of the human cerebral cortex has an overall average of 2.5-2.8 mm, usually varying between 1 and $5 \mathrm{~mm}$ (Makris et al., 2006). This measure may offer an adequate understanding of disease progression and possibly support clinical diagnosis and decision regarding treatment options (Hutton et al., 2008). Methods using CT are considered sensitive in the detection of structural abnormalities (Hutton et al., 2009), allowing for the opportunity to explore neural correlates of vulnerability to anxiety.

Changes in CT have been previously reported in normal and neurodegenerative development (e.g., Singh et al., 2006) and psychiatric disorders, such as major depression (for a review, see Suh et al., 2019). In relation to anxiety disorders, differences in CT across brain regions have been associated with individual differences in anxiety symptoms (Donzuso et al., 2014; Kühn et al., 2011). Nonetheless, scattered results are found across studies. For example, trait-anxiety has been negatively associated with $\mathrm{CT}$ in the right medial orbitofrontal cortex (mOFC; Kühn et al., 2011), but in another study significant effects were not found for this measure (Donzuso et al., 2014).

Rationale. Evidence from studies on functional and structural neural data support the identification of biomarkers of risk for the development of anxiety disorders (Blackmon et al., 2011) and discrepancies between studies. According to Pink et al. (2017), such discrepancies across studies may be due either to methodological differences or to other unknown mechanisms that may lie behind changes in CT and that are to date not fully understood.

Even though there has been a significant progress towards the development of the neural correlates of anxiety, to what extent changes in CT contribute to the development of anxiety disorders is still unclear. By conducting a scoping review aimed to scope the field of CT and its 
association with extant classic anxiety measures, we will have the opportunity to map the available literature, towards the identification of discrepancies and commonalities across studies.

Objectives. The objectives of the scoping review are: (a) to systematically identify and describe the nature of the available studies regarding the topic at hand (namely MRI pre-processing methods and data analysis used), (b) to summarize the main findings of the reviewed studies, and (c) to understand the association between CT and anxiety to better inform the mechanisms behind the development of anxiety disorders.

Consequently, the following research questions were formulated:

1. What are the most common methods used to estimate CT?

2. What are the most common methods or processes used to measure anxiety?

3. What is known from the literature regarding the association between $\mathrm{CT}$ and measures of anxiety or related processes (e.g., fear acquisition)?

4. What are the gaps in the current knowledge regarding the association between $\mathrm{CT}$ and anxiety?

Even though these are the central questions, the research team might add additional ones based on the process of searching and selecting the relevant studies, as scoping reviews are an iterative process.

\section{Methods}

Protocol and Study Design. The scoping review methodology, "a form of knowledge synthesis that addresses an exploratory research question aimed at mapping key concepts, types of evidence, and gaps in research related to a defined area or field by systematically searching, selecting and synthesizing existing knowledge" (Colquhoun et al., 2014, p. 1292-94), will be undertaken. The process of the scoping review will be guided by the Arksey and O'Malley (2005) methodology framework of six stages, further developed by Levac et al. (2010) and the Joanna Briggs Institute (Peters et al., 2017): (1) Identification of the research question(s), (2) Identification of relevant studies, (3) Selection of studies based on the inclusion and exclusion criteria, (4) Extraction and charting the data, (5) Collating, summarizing and reporting the findings, and (6) Expert consultation. The optional last phase will be undertaken and three experts on neuroimaging will be consulted once and asked to provide insights beyond what is reported in the literature.

The research team found the methodology of the scoping review more appropriate because of the broad research questions formulated, aiming to perform a comprehensive scope of the existing research and literature. 
This protocol was drafted using the guidelines and items of the Preferred Reporting Items for Systematic reviews and Meta-analyses extension for Scoping Reviews (PRISMA-ScR; Tricco et al., 2018).

Eligibility Criteria. This protocol will follow the PCC (Population, Concept and Context) mnemonic.

Population/Types of Participants. Participants should be human, young adults or adults (age range 18-60), with at least one clinical diagnosis of anxiety disorder (according to DSM-IV, DSM-IV-TR, DSM-5, and ICD-10) or healthy subjects. As anxiety and mood disorders often occur together, comorbidities between these two clinical conditions will be included. OCD, acute stress disorder and PTSD will be excluded due to not being currently considered anxiety disorders. Aging will be preliminarily excluded ( $>60$ years old), due to the expected and well-known negative correlations between age and CT in healthy aging (Salat et al., 2004), unless the studies control for age.

Concept. All CT methods and analysis will be included. The following anxiety-related measures will be included: clinical measures of self-report, psychophysiological measures (skin conductance response, heart rate variability), neurophysiological measures (fMRI), behavioral measures (avoidance), and measures of expectancy and learning of fear response. Genetic, epigenetic, neuroinflammatory, and neuroendocrinal measures are considered out of the scope of the current review.

Context. The research clinical setting can be pre-clinical, clinical or translational. Geographical location will not be limited.

Study Designs. Randomized controlled trials (RCTs), experimental studies, cohort studies, pre-clinical studies, and non-clinical studies will be included. Opinion articles, qualitative studies, and reviews (systematic reviews, meta-analysis, evidence maps, evidence syntheses) will be excluded.

Other. Only publications written in English or Portuguese will be considered for inclusion. Given the technological progress of neuroimaging, only studies from the last fifteen years will be selected (from 2004 to January 2020).

Information Sources. The present scoping review will include articles retrieved from the following electronic databases: PubMed, PsycINFO and PsycARTICLES, and Web of Science. In 
order to include all relevant articles in the scoping review, we will also check the reference lists of key studies.

Search Strategy. In accordance with the methodology for scoping reviews from the Joanna Briggs Institute (Peters et al., 2017), a three-step strategy will be utilized. Firstly, a search of the PubMed database will take place, with the terms "(cortical thickness AND anx*) OR (cortical thickness AND fear)". After this initial search, keywords searched in all fields of the retrieved articles will be analyzed, as well as the index terms. The next step will include a second search across databases, involving the identified key terms. Language and time frame limits will be applied. The program Rayyan (Ouzzani et al., 2016) will be used to manage the references. The searches will be conducted in January 2020.

Selection of Sources of Evidence. The selection process for the included sources of evidence will begin with the exclusion of duplicated articles across the databases. This process will consist of two steps, a title and abstract review and a full-text review. In the first step, two members of the research team will independently screen the title and abstracts of retrieved articles, checking if they are eligible for the next step, a full text retrieval. In the second step, the selected full-text articles will be analyzed, in terms of the inclusion and exclusion criteria. Inter-rater discrepancies/disagreements will be resolved through discussion until a consensus is reached. If the full consensus is not obtained, a third investigator will provide his input.

Data Charting Process and Data Items. The two reviewers will independently extract data from the eligible articles. A data-charting form will be developed a priori, based on characteristics of the study (authors, year of publication, study design, setting), methods (measures of anxiety, pre-processing methods, whole brain vs. region-of-interest, estimation of CT methods, method of statistical analysis, software used to process images, scanner strength, indication of manual editing for quality assurance, controlled variables), participants (sample size, age, sex, sample status [healthy or anxious], diagnosis included, medication status) and results (effect vs. no effect, direction of effect, brain regions implicated in significant results). After the comparison of each reviewer's charted data, disagreements will be resolved through discussion and a third reviewer as well.

Critical Appraisal of Individual Sources of Evidence. Since our scoping view aims to map the available literature and aggregate findings, we will perform a critical appraisal of individual sources of evidence but will consider all individual studies regardless its quality. To this end, we will use the Checklist for Analytical Cross-Sectional Studies of the Joanna Briggs Institute (Moola et al., 2020). 
Synthesis of Results. The findings will be presented through a tabular and narrative format and a PRISMA flow diagram will be used to report the results. Data analysis will then involve numeric and narrative approaches, in order to compare the included studies and find gaps in knowledge. The research team will address the gaps in the literature, as one of the main objectives of the scoping review.

\section{Discussion}

Implications. This scoping review will help design future studies and develop the understanding of the association between CT and anxiety.

Dissemination. An article reporting the process and results of the scoping review will be submitted to journals with peer review and impact factor, Q1 or Q2.

Strengths of the Study. The research team will conduct a comprehensive and broad literature search of several electronic databases, and a quality assessment of the reviewed articles will be performed.

Limitations of the Study. The search will be limited to English and Portuguese languages, and the scope of the review does not include a comprehensive approach to all anxiety measures, leaving behind a few innovative measures (e.g. neuroendocrine, genetic, and epigenetic biomarkers).

\section{References}

American Psychiatric Association. (2013). Diagnostic and statistical manual of mental disorders (5th ed.). https://doi.org/10.1176/appi.books.9780890425596

Arksey, H., \& O’Malley, L. (2005). Scoping studies: Towards a methodological framework. International Journal of Social Research Methodology, 8(1), 19-32. https://doi.org/10.1080/1364557032000119616

Bandelow, B., \& Michaelis, S. (2015). Epidemiology of anxiety disorders in the 21st century. Dialogues in Clinical Neuroscience, 17(3), 327-335.

Beck, A. T., Epstein, N., Brown, G., \& Steer, R. A. (1988). An inventory for measuring clinical anxiety: Psychometric properties. Journal of Consulting and Clinical Psychology, 56(6), 893-897. https://doi.org/10.1037/0022-006X.56.6.893 
Blackmon, K., Barr, W. B., Carlson, C., Devinsky, O., DuBois, J., Pogash, D., Quinn, B. T., Kuzniecky, R., Halgren, E., \& Thesen, T. (2011). Structural evidence for involvement of a left amygdala-orbitofrontal network in subclinical anxiety. Psychiatry Research: Neuroimaging, 194(3), 296-303. https://doi.org/10.1016/j.pscychresns.2011.05.007

Colquhoun, H. L., Levac, D., O'Brien, K. K., Straus, S., Tricco, A. C., Perrier, L., Kastner, M., \& Moher, D. (2014). Scoping reviews: Time for clarity in definition, methods, and reporting. Journal of Clinical Epidemiology, 67(12), 1291-1294. https://doi.org/10.1016/j.jclinepi.2014.03.013

Das, S. R., Avants, B. B., Grossman, M., \& Gee, J. C. (2009). Registration based cortical thickness measurement. $\quad$ NeuroImage, $\quad 45(3), \quad 867-879$. https://doi.org/10.1016/j.neuroimage.2008.12.016

Donzuso, G., Cerasa, A., Gioia, M. C., Caracciolo, M., \& Quattrone, A. (2014). The neuroanatomical correlates of anxiety in a healthy population: Differences between the State-Trait Anxiety Inventory and the Hamilton Anxiety Rating Scale. Brain and Behavior, 4(4), 504-514. https://doi.org/10.1002/brb3.232

Gold, A. L., Steuber, E. R., White, L. K., Pacheco, J., Sachs, J. F., Pagliaccio, D., Berman, E., Leibenluft, E., \& Pine, D. S. (2017). Cortical thickness and subcortical gray matter volume in pediatric anxiety disorders. Neuropsychopharmacology, 42(12), 2423-2433. https://doi.org/10.1038/npp.2017.83

Hamilton, M. (1959). The assessment of anxiety states by rating. The British Journal of Medical Psychology, 32(1), 50-55. https://doi.org/10.1111/j.2044-8341.1959.tb00467.x

Hutton, C., Draganski, B., Ashburner, J., \& Weiskopf, N. (2009). A comparison between voxelbased cortical thickness and voxel-based morphometry in normal aging. NeuroImage, 48(2), 371-380. https://doi.org/10.1016/j.neuroimage.2009.06.043

Hutton, C., Vita, E. D., Ashburner, J., Deichmann, R., \& Turner, R. (2008). Voxel-based cortical thickness measurements in MRI. NeuroImage, 40(4), 1701-1710. https://doi.org/10.1016/j.neuroimage.2008.01.027

Kühn, S., Schubert, F., \& Gallinat, J. (2011). Structural correlates of trait anxiety: Reduced thickness in medial orbitofrontal cortex accompanied by volume increase in nucleus accumbens. Journal of Affective Disorders, 134(1-3), 315-319. https://doi.org/10.1016/j.jad.2011.06.003 
Levac, D., Colquhoun, H., \& O’Brien, K. K. (2010). Scoping studies: Advancing the methodology. Implementation Science, 5, Article 69. https://doi.org/10.1186/1748-5908-5-69

Makris, N., Kaiser, J., Haselgrove, C., Seidman, L. J., Biederman, J., Boriel, D., Valera, E. M., Papadimitriou, G. M., Fischl, B., Caviness, V. S., \& Kennedy, D. N. (2006). Human cerebral cortex: A system for the integration of volume- and surface-based representations. NeuroImage, 33(1), 139-153. https://doi.org/10.1016/j.neuroimage.2006.04.220

Moola, S., Munn, Z., Tufanaru, C., Aromataris, E., Sears, K., Sfetcu, R., Currie, M., Lisy, K., Qureshi, R., Mattis, P., \& Mu, P. (2020). Chapter 7: Systematic reviews of etiology and risk. In E. Aromataris \& Z. Munn (Eds.), JBI Manual for Evidence Synthesis (pp. 219-272). The Joanna Briggs Institute. https://doi.org/10.46658/JBIMES-20-08

Ouzzani, M., Hammady, H., Fedorowicz, Z., \& Elmagarmid, A. (2016). Rayyan - A web and mobile app for systematic reviews. Systematic Reviews, 5(1), Article 210. https://doi.org/10.1186/s13643-016-0384-4

Peters, M. D. J., Godfrey, C., McInerney, P., Soares, C. B., Khalil, H., \& Parker, D. (2017). Chapter 11: Scoping Reviews. In The Joanna Briggs Institute Reviewers' Manual 2015. The Joanna Briggs Institute.

Pink, A., Przybelski, S. A., Krell-Roesch, J., Stokin, G. B., Roberts, R. O., Mielke, M. M., Spangehl, K. A., Knopman, D. S., Jack, C. R. J., Petersen, R. C., \& Geda, Y. E. (2017). Cortical thickness and anxiety symptoms among cognitively normal elderly persons: The mayo clinic study of aging. The Journal of Neuropsychiatry and Clinical Neurosciences, 29(1), 60-66. https://doi.org/10.1176/appi.neuropsych.15100378

Salat, D. H., Buckner, R. L., Snyder, A. Z., Greve, D. N., Desikan, R. S. R., Busa, E., Morris, J. C., Dale, A. M., \& Fischl, B. (2004). Thinning of the cerebral cortex in aging. Cerebral Cortex, 14(7), 721-730. https://doi.org/10.1093/cercor/bhh032

Shin, L. M., \& Liberzon, I. (2010). The neurocircuitry of fear, stress, and anxiety disorders. Neuropsychopharmacology, 35(1), 169-191. https://doi.org/10.1038/npp.2009.83

Singh, V., Chertkow, H., Lerch, J. P., Evans, A. C., Dorr, A. E., \& Kabani, N. J. (2006). Spatial patterns of cortical thinning in mild cognitive impairment and Alzheimer's disease. Brain, 129(11), 2885-2893. https://doi.org/10.1093/brain/awl256 
Spielberger, C. D., Gorsuch, R. L., Lushene, R., Vagg, P. R., \& Jacobs, G. A. (1983). Manual for the State-Trait Anxiety Inventory. Consulting Psychologists Press.

Suh, J. S., Schneider, M. A., Minuzzi, L., MacQueen, G. M., Strother, S. C., Kennedy, S. H., \& Frey, B. N. (2019). Cortical thickness in major depressive disorder: A systematic review and meta-analysis. Progress in Neuro-Psychopharmacology and Biological Psychiatry, 88 , 287-302. https://doi.org/10.1016/j.pnpbp.2018.08.008

Tinoco-González, D., Fullana, M. A., Torrents-Rodas, D., Bonillo, A., Vervliet, B., Blasco, M. J., Farré, M., \& Torrubia, R. (2015). Conditioned fear acquisition and generalization in generalized anxiety disorder. Behavior Therapy, 46(5), 627-639. https://doi.org/10.1016/j.beth.2014.12.004

Tricco, A. C., Lillie, E., Zarin, W., O’Brien, K. K., Colquhoun, H., Levac, D., Moher, D., Peters, M. D. J., Horsley, T., Weeks, L., Hempel, S., Akl, E. A., Chang, C., McGowan, J., Stewart, L., Hartling, L., Aldcroft, A., Wilson, M. G., Garritty, C., ... Straus, S. E. (2018). PRISMA extension for scoping reviews (PRISMA-ScR): Checklist and explanation. Annals of Internal Medicine, 169(7), 467-473. https://doi.org/10.7326/M18-0850 


\section{Appendix B}

Identified Key Terms Across the Databases

\begin{tabular}{|c|c|}
\hline Database & Key terms \\
\hline Web of Science & $\begin{array}{l}\mathrm{TS}=((\text { cortical thickness }) \text { AND (anx* OR anxiety disorders) AND (mri OR } \\
\text { anatomical) }) \text { OR TS }=((\text { cortical thickness }) \text { AND (fear acquisition OR fear } \\
\text { extinction OR fear conditioning OR fear }) \text { AND (mri OR anatomical }))\end{array}$ \\
\hline PubMed & $\begin{array}{l}\text { ((“cortical thickness") AND (anx* OR anxiety disorders) AND (mri OR } \\
\text { anatomical)) OR ((“cortical thickness") AND (fear acquisition OR fear } \\
\text { extinction OR fear conditioning OR fear) AND (mri OR anatomical)) }\end{array}$ \\
\hline \multirow{5}{*}{$\begin{array}{l}\text { PsycINFO and } \\
\text { PsycARTICLES }\end{array}$} & \# Searches \\
\hline & 1 (cortical thickness and (anx* or anxiety disorders) and (mri or anatomical)).mp. [mp=ti, ab, tx, ct, hw, tc, id, ot, tm, mhl] \\
\hline & 2 limit 1 to y=2004-Current \\
\hline & 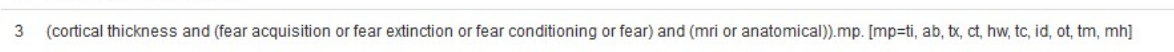 \\
\hline & 41 or 3 \\
\hline
\end{tabular}




\section{Appendix C}

\section{Final Search Strategy for the PsycINFO Database}

Database: APA PsycArticles Full Text, APA PsycInfo <2004 to January Week 2 2020> Search Strategy:

1 (cortical thickness and (anx* or anxiety disorders) and (mri or anatomical)).mp. [mp=ti, ab, tx, ct, hw, tc, id, ot, tm, mh]

2 (cortical thickness and (fear acquisition or fear extinction or fear conditioning or fear) and (mri or anatomical)).mp. [mp=ti, ab, tx, ct, hw, tc, id, ot, tm, mh]

31 or 2 


\section{Appendix D}

\section{Joanna Briggs Institute Critical Appraisal Checklist for Analytical Cross-Sectional Studies}

Reviewer Date

Author Year Record Number

Yes No Unclear $\begin{gathered}\text { Not } \\ \text { applicable }\end{gathered}$

1. Were the criteria for inclusion in the sample clearly defined?

2. Were the study subjects and the setting described in detail?

3. Was the exposure measured in a valid and reliable way?

4. Were objective, standard criteria used for measurement of the condition?

5. Were confounding factors identified?

6. Were strategies to deal with confounding factors stated?

7. Were the outcomes measured in a valid and reliable way?

8. Was appropriate statistical analysis used?

Overall appraisal: Include $\square$ Exclude $\square$ Seek further info Comments (Including reason for exclusion)

\section{Reference}

Moola, S., Munn, Z., Tufanaru, C., Aromataris, E., Sears, K., Sfetcu, R., Currie, M., Lisy, K., Qureshi, R., Mattis, P., \& Mu, P. (2020). Chapter 7: Systematic reviews of etiology and risk. In E. Aromataris \& Z. Munn (Eds.), Joanna Briggs Institute Reviewer's Manual (pp. 219-272). The Joanna Briggs Institute. https://doi.org/10.46658/JBIRM-19-01 


\section{Appendix E}

\section{Critical Appraisal of Individual Sources of Evidence}

\begin{tabular}{|c|c|c|c|c|c|c|c|c|}
\hline Study & Item 1 & Item 2 & Item 3 & Item 4 & Item 5 & Item 6 & Item 7 & Item 8 \\
\hline Asami et al. (2018) & $\mathrm{Yes}^{\mathrm{a}}$ & Yes & Yes & Yes & Yes & Yes & Yes & Yes \\
\hline Blackmon et al. (2011) & Yes & Yes & Yes & No & Yes & Yes & Yes & Yes \\
\hline Brühl et al. (2014) & Yes & Yes & Yes & Yes & Unclear & Unclear & Yes & Yes \\
\hline Carnevali et al. (2019) & Yes & Yes & Yes & Yes & Yes & Yes & Yes & Yes \\
\hline Cha et al. (2014) & No & Yes & Yes & Yes & Yes & Yes & Yes & Yes \\
\hline Donzuso et al. (2014) & Yes & Yes & Yes & Yes & Yes & Yes & Yes & Yes \\
\hline Hartley et al. (2011) & Unclear & Unclear & Yes & Unclear & Unclear & Unclear & Yes & Yes \\
\hline Kang et al. (2017) & Yes & Yes & Yes & Yes & Yes & Yes & Yes & Yes \\
\hline Kühn et al. (2011) & Yes & Yes & Yes & Yes & Yes & Yes & Yes & Yes \\
\hline Maggioni et al. (2019) & Yes & Yes & Yes & Yes & Yes & Yes & Yes & Yes \\
\hline Milad et al. (2005) & Unclear & $\mathrm{No}^{\mathrm{b}}$ & Yes & Unclear & No & No & Yes & Yes \\
\hline Milad et al. (2007) & Unclear $^{\mathrm{a}}$ & No & Yes & Unclear & No & No & Yes & Yes \\
\hline Molent et al. (2018) & Yes & Yes & Yes & Yes & Yes & Yes & Yes & Yes \\
\hline Rosso et al. (2010) & Yes & Yes & Yes & Yes & Yes & Yes & Yes & Yes \\
\hline Syal et al. (2012) & Unclear & Yes & Yes & Yes & Yes & Yes & Yes & Yes \\
\hline Zhao et al. (2017) & Yes & Yes & Yes & Yes & Yes & Yes & Yes & Yes \\
\hline Winkelmann et al. (2016) & Unclear & Yes & Yes & Yes & Yes & Yes & Yes & Yes \\
\hline
\end{tabular}

Note. The following questions correspond to the items: Item 1 ("Were the criteria for inclusion in the sample clearly defined?"), Item 2 ("Were the study subjects and setting described in detail?"), Item 3 ("Was the exposure [cortical thickness] measured in a valid and reliable way?"), Item 4 (“Were objective, standard criteria used for measurement of the condition [anxiety disorders or absence of disorder]?”), Item 5 (“Were cofounding factors identified?"), Item 6 (“Were strategies to deal with cofounding factors stated?"), Item 7 (“Were the outcomes [anxiety-related measures] measured in a valid and reliable way?"), and Item 8 ("Was appropriate statistical analysis used?"). Possibility of answers include "Yes", "No", "Unclear" and "Not Applicable".

a The exclusion/inclusion criteria were reported in a previous study. 